\title{
Evaluation of the Use of Existing RELAP5-3D Models to Represent the Actinide Burner Test Reactor
}

C.B. Davis

February 2007

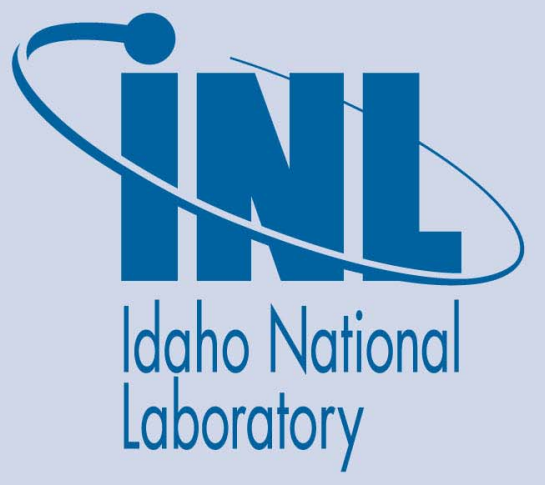

The INL is a U.S. Department of Energy National Laboratory operated by Battelle Energy Alliance 


\title{
Evaluation of the Use of Existing RELAP5-3D Models to Represent the Actinide Burner Test Reactor
}

\author{
C.B. Davis
}

February 2007

Idaho National Laboratory
Idaho Falls, Idaho 83415

http://www.inl.gov

Prepared for the

U.S. Department of Energy

Office of Nuclear Energy

Under DOE Idaho Operations Office

Contract DE-AC07-05ID14517 


\begin{abstract}
The RELAP5-3D code is being considered as a thermal-hydraulic system code to support the development of the sodium-cooled Actinide Burner Test Reactor as part of Global Nuclear Energy Partnership. An evaluation was performed to determine whether the control system could be used to simulate the effects of non-convective mechanisms of heat transport in the fluid that are not currently represented with internal code models, including axial and radial heat conduction in the fluid and subchannel mixing. The evaluation also determined the relative importance of axial and radial heat conduction and fluid mixing on peak cladding temperature for a wide range of steady conditions and during a representative loss-of-flow transient. The evaluation was performed using a RELAP5-3D model of a subassembly in the Experimental Breeder Reactor-II, which was used as a surrogate for the Actinide Burner Test Reactor. An evaluation was also performed to determine if the existing centrifugal pump model could be used to simulate the performance of electromagnetic pumps.
\end{abstract}




\section{SUMMARY}

The Actinide Burner Test Reactor (ABTR) is envisioned as a sodium-cooled, fast reactor that will burn the actinides generated in light water reactors to reduce nuclear waste and ease proliferation concerns as part of the Global Nuclear Energy Partnership. The RELAP5-3D code is being considered as a thermalhydraulic system code to support the development of the ABTR. An evaluation of the code's applicability for modeling the ABTR indicated that the lack of models for heat conduction and mixing in the fluid and electromagnetic pumps could be important. Consequently, an evaluation was performed to determine whether existing models in the code could be used to simulate the performance of the electromagnetic pumps and the non-convective mechanisms of energy transport in the fluid, which include heat conduction and subchannel mixing. The evaluation also determined the relative importance of axial and radial heat conduction in the fluid and radial mixing between rings within a subassembly on cladding temperature for a wide range of steady-state conditions and during a representative loss-of-flow transient. The evaluation was performed using a RELAP5-3D model of a subassembly in the Experimental Breeder Reactor-II, which was used as a surrogate for the ABTR.

The evaluation showed that the effects of heat conduction in the fluid and radial mixing could be simulated using the RELAP5-3D control system. However, it would be far easier from the user's perspective to represent these phenomena with an internal code model that could be turned on or off rather than with the control system. Because of the finite number of available control variables, the number of junctions at which conduction and mixing can be represented with the control system is limited to about 430. Internal models should be added to the code to simulate these phenomena so that the analysis of the ABTR is not limited by the number of available control variables.

The evaluation concluded that the effects of axial conduction in the fluid were generally not important. The steady-state calculations showed that axial conduction did not noticeably affect the fluid temperature profile in the core until the normalized power and flow decreased to about $0.1 \%$ of the design values. The effects of axial conduction were large when the normalized power and flow decreased to $0.01 \%$ of the design values. Even though the normalized flow during the loss-of-flow event was less than $0.1 \%$ of the design value for more than $40 \mathrm{~s}$, transient terms dominated and axial conduction did not significantly affect the peak cladding temperature or the fluid temperature profile in the core. The effects of axial conduction in the fuel rods and other subassembly heat structures were smaller than those due to axial conduction in the fluid.

The evaluation concluded that subchannel effects within the subassembly are important in the calculation of peak cladding temperature in the ABTR. Two-dimensional RELAP5-3D models predicted more than $100 \mathrm{~K}$ radial variations in fluid temperature at the top of the core during normal operation, primarily because the outermost ring was bounded by an unheated subassembly wall. A one-dimensional model underestimated the maximum fluid temperature in the subassembly by more than $50 \mathrm{~K}$ during normal operation. The one-dimensional model did not account for the internal recirculation due to buoyancy and thus neglected an important cooling mechanism during the loss-of-flow transient. As a result, the peak cladding temperature obtained with the one-dimensional model was at least $130 \mathrm{~K}$ higher than the corresponding values from the two-dimensional models during the loss-of-flow transient.

The evaluation concluded that the effects of radial conduction in the fluid were important. The steadystate analyses showed that radial conduction did not significantly affect the maximum fluid temperature in the subassembly, but significantly affected the radial temperature profile when the normalized flow and power were $10 \%$ or more of the design values. The effects of radial conduction on maximum fluid temperature were small when the normalized flow and power were $1 \%$ or less because of internal subassembly flow due to buoyancy. Radial conduction in the fluid significantly affected the peak 
cladding temperature during the loss-of-flow transient. A two-dimensional calculation with radial conduction was $64 \mathrm{~K}$ higher than a two-dimensional calculation without radial conduction.

Radial mixing in a subassembly, which is caused by a combination of the flow induced by the wire wrapping and turbulence, was also important at high flow rates. The steady-state analyses showed that the mixing between subassemblies significantly affected the radial fluid temperature profile when the normalized flow and power were $10 \%$ or more of the design values. The effect of mixing on the peak cladding temperature during a loss-of-flow transient was small because the amount of mixing is proportional to the axial flow rate, which was small during the event.

The evaluation concluded that the performance of an electromagnetic pump could be adequately represented with the code's centrifugal pump model. The homologous curves used in the centrifugal pump model adequately represented the performance predicted by a mechanistic electromagnetic pump model during normal operation. Homologous curves also reasonably represented the performance of a simple, linear model during a transient. 


\section{CONTENTS}

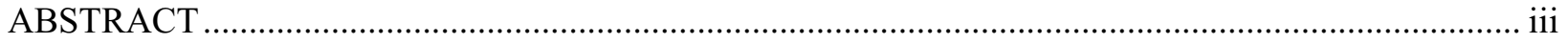

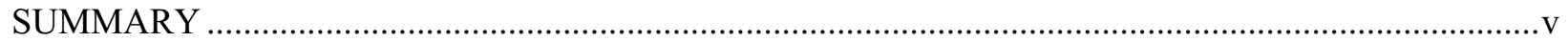

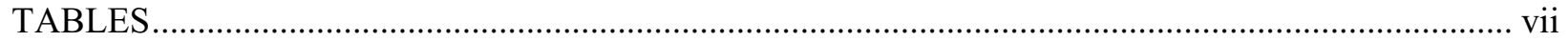

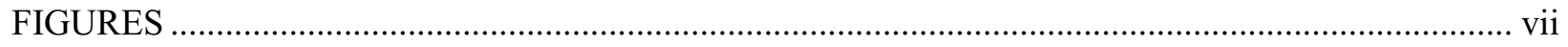

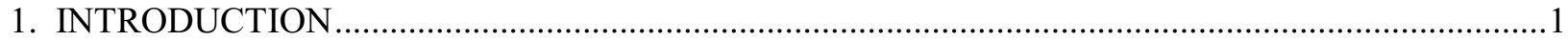

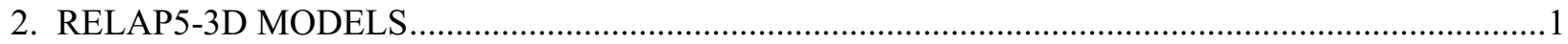

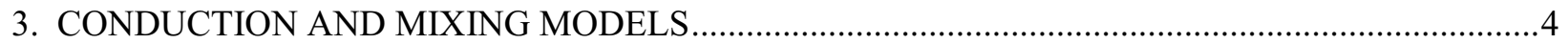

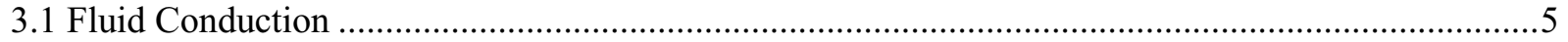

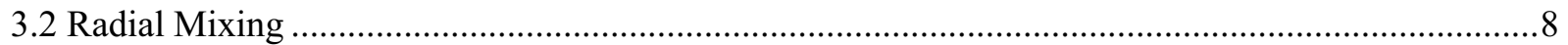

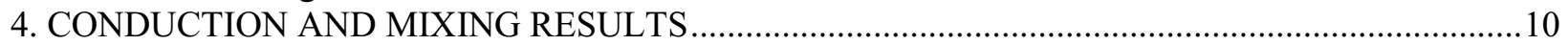

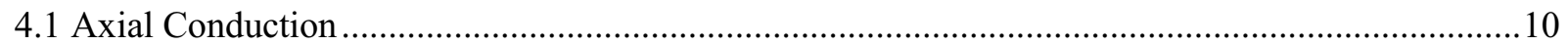

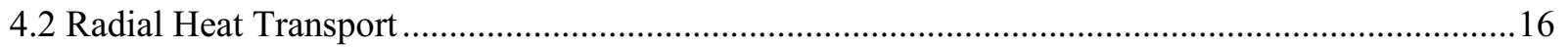

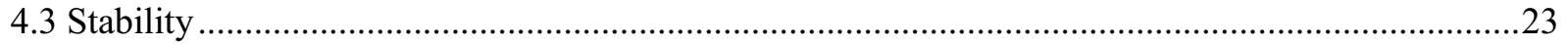

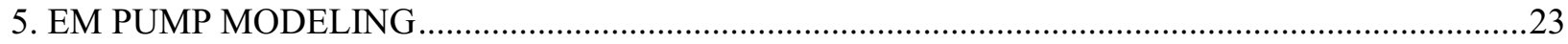

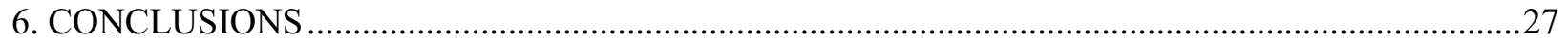

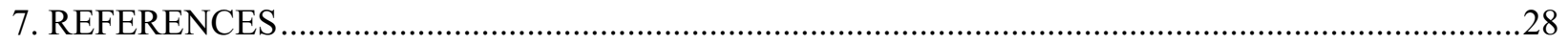

\section{TABLES}

1. Rated parameters for the EM pump modeled by Murray (2005) .................................................. 25

\section{FIGURES}

1. Schematic of the XX09 subassembly (from Messick et al. 1987) .................................................... 2

2. Cross-sectional view of the XX09 subassembly (from Messick et al. 1987) ..................................... 2

3. One-dimensional RELAP5-3D model of the XX09 subassembly ...................................................... 3

4. Two-dimensional RELAP5-3D model of the XX09 subassembly ................................................... 4

5. Nodalization diagram for the axial fluid heat conduction model ....................................................... 6

6. Nodalization diagram for the axial fluid heat conduction model with multiple junctions

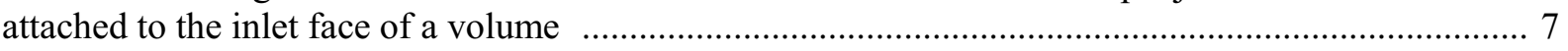

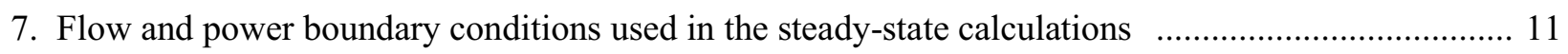

8. Modified Peclet numbers at the center of the core in the steady-state calculations ............................ 11

9. Axial fluid temperature profiles for the cases without axial conduction ........................................ 12

10. Axial fluid temperature profiles for the cases with axial conduction in the fluid ............................ 12

11. Axial fluid temperature profiles for the cases with axial conduction in the fluid

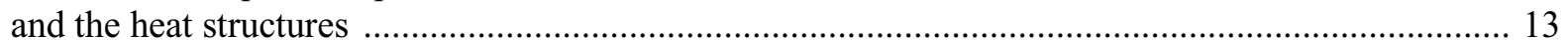

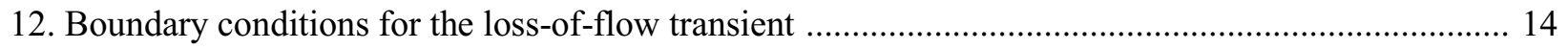

13. Maximum cladding temperature during the loss-of-flow transient ............................................... 15

14. Axial fluid temperature profiles during the loss-of-flow transient ............................................ 15

15. Steady-state radial fluid temperature profiles at the top of the core (without radial heat transport) ... 17

16. Steady-state radial fluid temperature profiles at the top of the core (with radial conduction) ............ 18

17. Steady-state radial fluid temperature profiles at the top of the core (with radial mixing) .................. 18

18. The effects of various models on steady-state radial fluid temperature profiles at the top of the core for a normalized flow and power of $100 \%$

19. The effects of various models on steady-state radial fluid temperature profiles at the top of the core for a normalized flow and power of $10 \%$ 
20. The effects of various models on steady-state radial fluid temperature profiles at the top of the core for a normalized flow and power of $1 \%$

21. The effects of various models on steady-state axial fluid velocity profiles at the top of the core for a normalized flow and power of $100 \%$

22. The effects of various models on steady-state axial fluid velocity profiles at the top of the core for a normalized flow and power of $1 \%$

23. The effects of radial heat transport mechanisms on the maximum cladding temperature during the loss-of-flow transient

24. The effects of radial heat transport mechanisms on the axial fluid velocity at the top of the

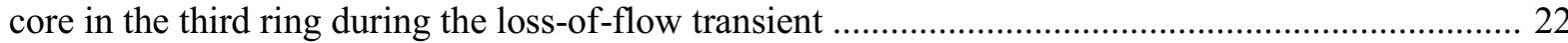

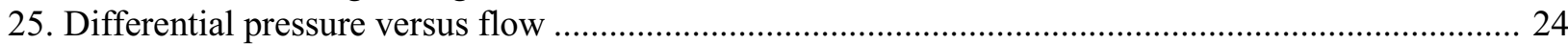

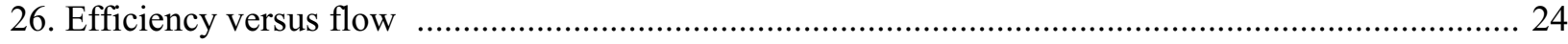

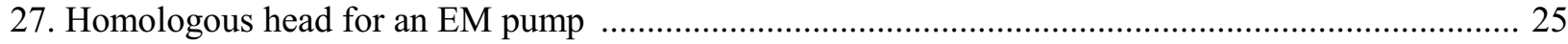

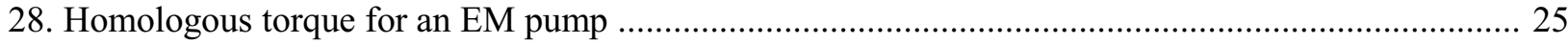

29. A comparison of linear and homologous EM pump models ......................................................... 27 


\section{INTRODUCTION}

The Actinide Burner Test Reactor (ABTR) is envisioned as a sodium-cooled, fast reactor that will burn the actinides generated in light water reactors to reduce nuclear waste and ease proliferation concerns as part of the Global Nuclear Energy Partnership. The RELAP5-3D (RELAP5-3D Development Team 2005) code is being considered as a thermal-hydraulic system code to support the development of the ABTR. An evaluation of the code's applicability for modeling the ABTR (Davis 2006) indicated that non-modeled mechanisms of heat transport, including heat conduction and mixing in the fluid, and the lack of models for electromagnetic (EM) pumps could be important.

An evaluation was performed to determine whether existing models in RELAP5-3D could be used to simulate the performance of the EM pumps and the non-modeled mechanisms of heat transport. The evaluation determined the importance of axial and radial heat conduction in the fluid for a wide range of steady-state conditions and during a representative loss-of-flow transient. The evaluation also determined the importance of mixing between adjacent rings within a subassembly. The evaluation used a RELAP53D model of a subassembly in the Experimental Breeder Reactor-II (EBR-II) because the ABTR has not yet been designed.

The figure of merit for evaluating the safety of the EBR-II was the peak temperature at the interface between the fuel and the cladding (Lehto et al. 1987). The temperature of the inner surface of the cladding was limited to $715{ }^{\circ} \mathrm{C}$ during normal operation and during anticipated transients. Messick et al. (1987) reported a maximum measured fluid temperature at the top of the core of about $550{ }^{\circ} \mathrm{C}$. Allowing for a $20^{\circ} \mathrm{C}$ temperature rise across the cladding, the margin between the maximum operating temperature and the safety limit was approximately $715-550-20=145^{\circ} \mathrm{C}$. The evaluations of the importance of heat conduction and mixing in the fluid were based on quantitative comparisons between the calculated effects and a value of $15^{\circ} \mathrm{C}$, which corresponds to about $10 \%$ of the margin during normal operation. Effects less than $15{ }^{\circ} \mathrm{C}$ were judged to be not important whereas effects greater than $15^{\circ} \mathrm{C}$ were judged to be important. The $15^{\circ} \mathrm{C}$ value used here is roughly consistent with values previously used by the Nuclear Regulatory Commission (NRC). For example, 10 CFR 50.46 (NRC 2007), which addresses acceptance criteria for emergency core cooling systems in light water reactors, defines a significant change as one that exceeds $28{ }^{\circ} \mathrm{C}$ in peak cladding temperature. The use of a smaller value for determining significance seems appropriate here because the safety limit allowed in EBR-II is much less than that allowed in light water reactors $\left(1204^{\circ} \mathrm{C}\right)$.

Section 2 of this report describes the EBR-II subassembly and the RELAP5-3D models used in the analysis. Section 3 describes the models for calculating the heat transport mechanisms associated with heat conduction in the fluid and radial mixing within a subassembly. Section 4 presents results from the evaluations of these heat transport mechanisms. Section 5 describes the evaluation for modeling the performance of EM pumps with RELAP5-3D. Conclusions and references are presented in Sections 6 and 7 , respectively.

\section{RELAP5-3D MODELS}

Two RELAP5-3D models were used in this evaluation. A one-dimensional model was used to simulate the effects of axial heat conduction in the fluid. A two-dimensional model was used to simulate the effects of radial conduction and mixing. 
The RELAP5-3D models were based on a model (Talley 2006) of the instrumented XX09 subassembly in EBR-II (Messick et al. 1987). The XX09 subassembly is illustrated in Figures 1 and 2. The subassembly contained 61 rods arranged in a triangular array within a hexagonal wall. The array contained 59 wirewrapped fuel rods and two rods that were used for instrument leads. Thermocouples were used to measure fluid temperatures near the top of the active core and in the mixing section near the top of the subassembly. The fuel rods contained a long gas plenum above the top of the core to accommodate fission gas release. The "annular" thimble flow region outside of the subassembly wall provided space for control rod insertion.

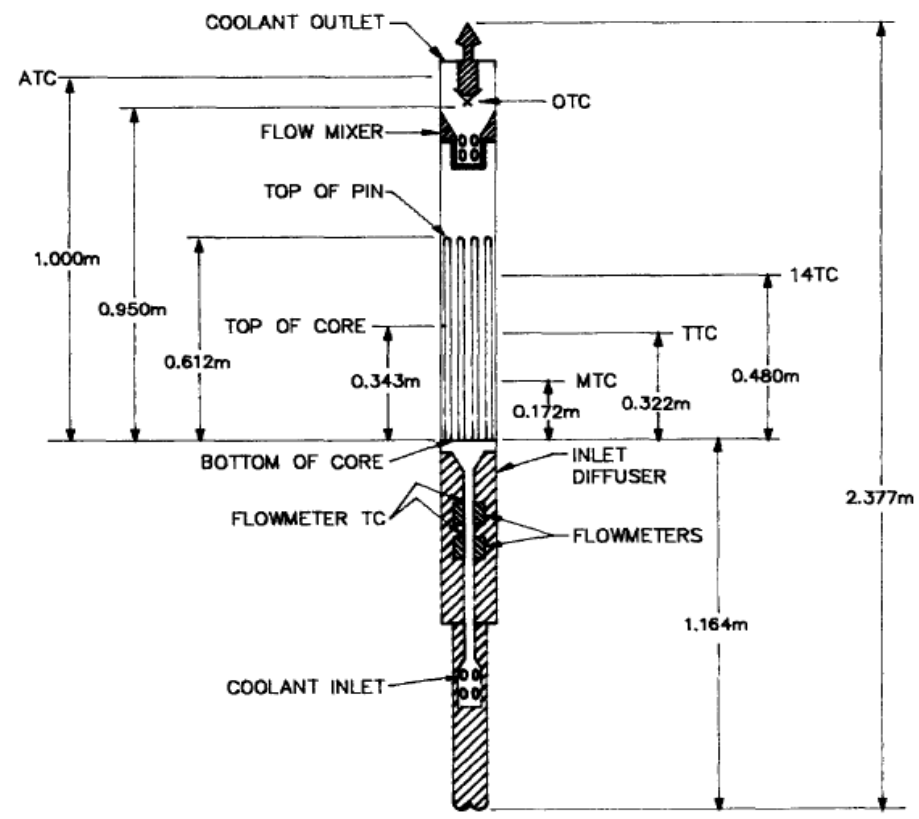

Figure 1. Schematic of the XX09 subassembly (from Messick et al. 1987).

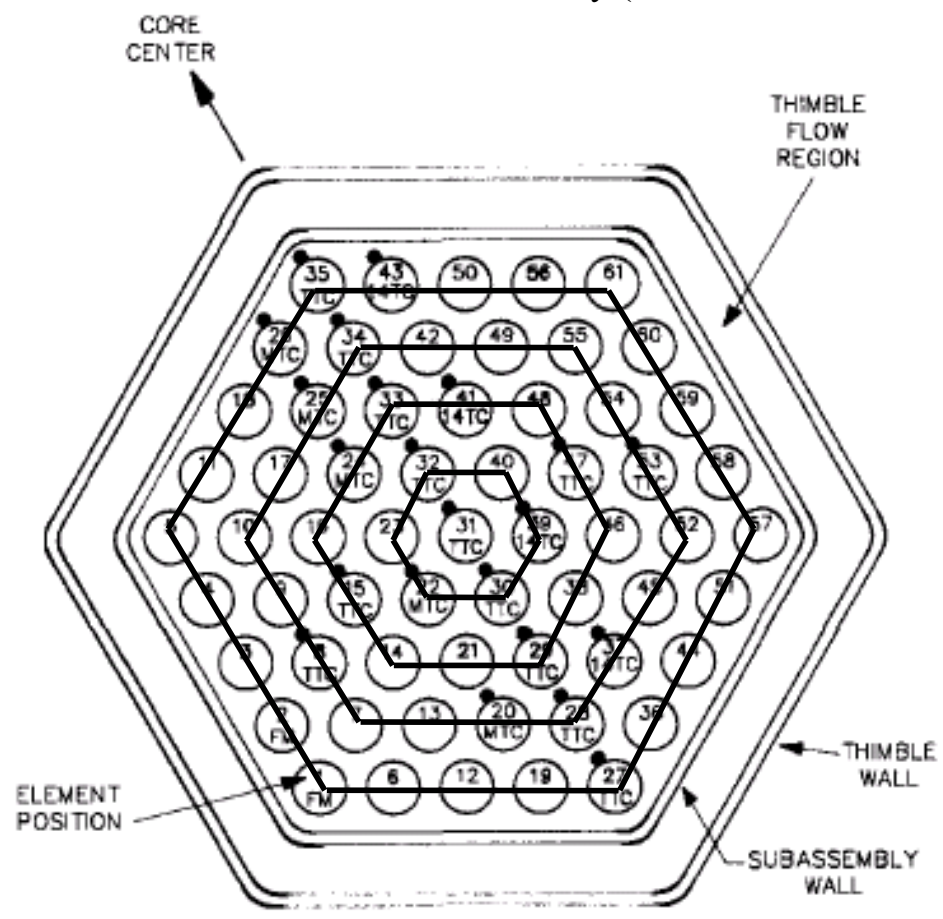

Figure 2. Cross-sectional view of the XX09 subassembly (from Messick et al. 1987). 
The one-dimensional model of the XX09 subassembly is illustrated in Figure 3. The model represented the inlet, active core, gas plenum, thimble, and outlet regions of the subassembly. The active core was modeled with Component 140, a one-dimensional pipe that contained ten axial control volumes. Heat structures were used to represent the fuel rods and the subassembly and thimble walls. Components 100 and 220 were time-dependent volumes that specified the temperature of the sodium entering the subassembly and the pressure at the top of the subassembly. Flow boundary conditions were applied at the bottom of the subassembly with a time-dependent junction (Component 110). The flow area at Junction 190 was adjusted so that the thimble received 5\% of the total flow at normal operating conditions. Messick et al. (1987) did not report the magnitude of the thimble flow, but Dunn et al. (2006) stated that it was small. The power applied to the fuel rods in the core was specified using a chopped cosine power profile with a maximum axial peaking factor of 1.2. The radial power distribution was assumed to be uniform within the subassembly. The outer surface of the thimble wall was assumed to be adiabatic.

The RELAP5-3D model does not represent the XX09 subassembly exactly because of a lack of information about the power and flow distributions within the XX09 and adjacent subassemblies and the geometry in the inlet and outlet regions. Nonetheless, it is considered representative of a fuel subassembly in a sodium-cooled fast reactor.

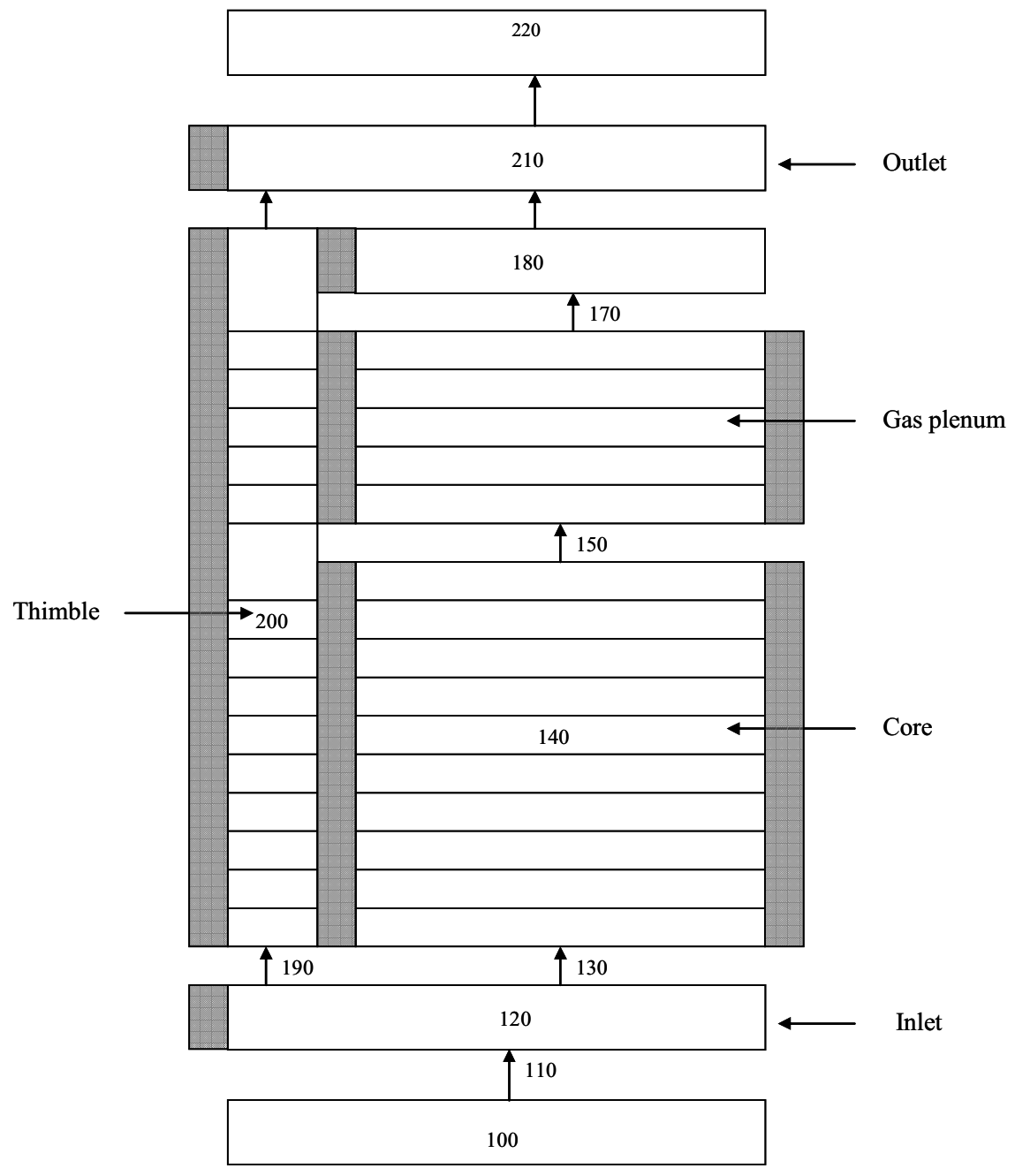

Figure 3. One-dimensional RELAP5-3D model of the XX09 subassembly. 
A two-dimensional RELAP5-3D model of the XX09 subassembly was also developed. The twodimensional model was identical to the one-dimensional model except that it used five parallel components to represent the core and gas plenum regions as shown in Figure 4. Each component represented one of the five hexagonal rings inside the subassembly wall shown in Figure 2. The boundaries between rings were defined by lines drawn through the center of each row of fuel rods. The five radial rings were represented by Components 141 through 145 in the core and Components 161 through 165 in the gas plenum region. Components 141 and 161 represented the first (innermost) ring, Components 142 and 162 represented the second ring, and so forth. Multiple junction components (Components 156 through 159 and 176 through 179) allowed crossflow between adjacent rings in the core and gas plenum regions.

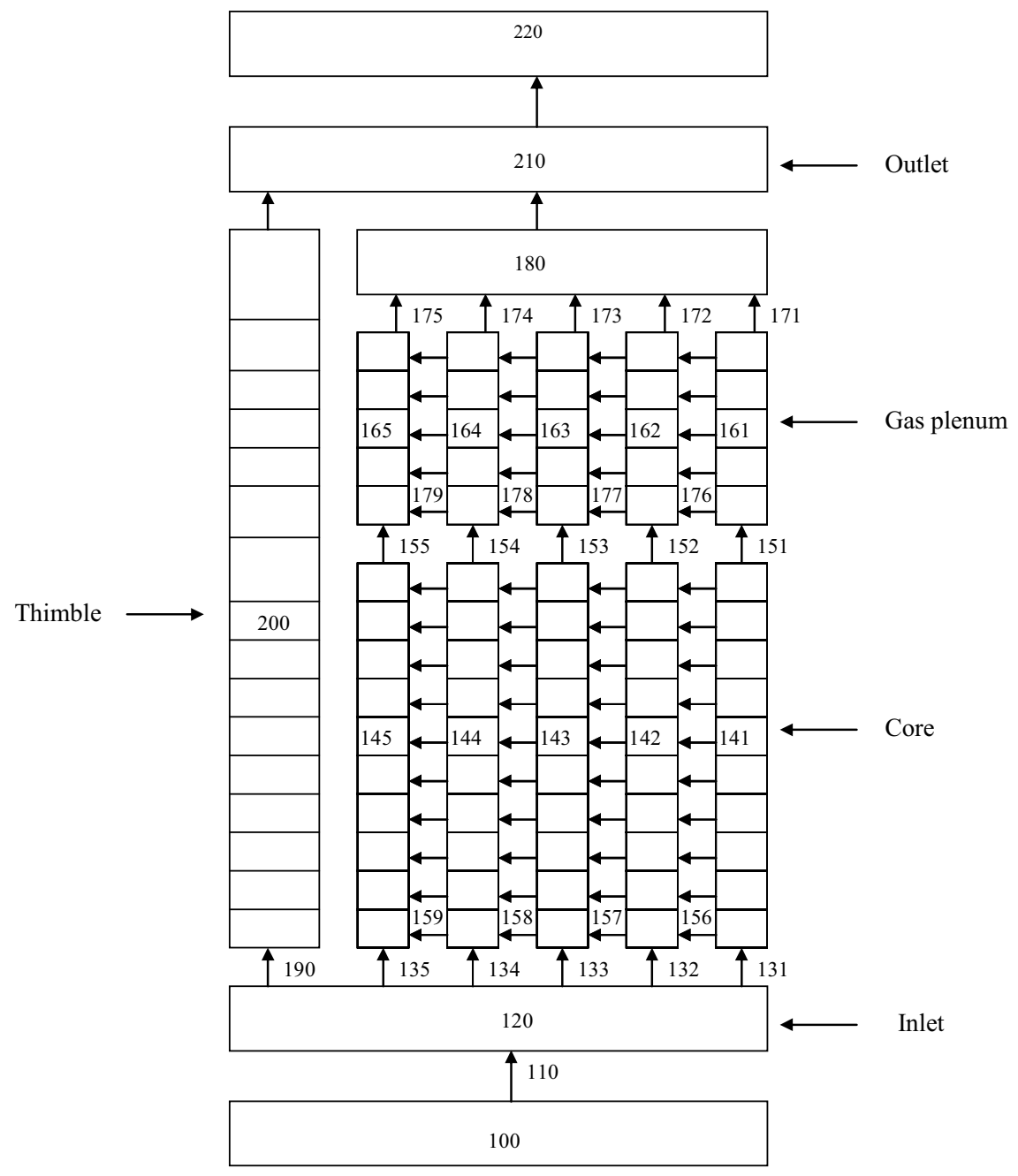

Figure 4. Two-dimensional RELAP5-3D model of the XX09 subassembly.

\section{CONDUCTION AND MIXING MODELS}

The control system model of RELAP5-3D was used to simulate the effects of fluid heat conduction and mixing. The control system provides a generalized capability to evaluate algebraic and differential equations using standard mathematical operations and functions that can interact with the code's 
hydrodynamic calculations. In this evaluation, the control system used fluid temperatures to calculate the heat transfer associated with heat conduction and radial mixing in the fluid. The calculated amount of heat was then added to or removed from the various control volumes in the subassembly to represent these heat transport mechanisms. The model used to represent fluid conduction is described in Section 3.1. The model was initially developed for axial conduction and then extended to represent radial conduction. Section 3.2 describes the development of the mixing model.

\subsection{Fluid Conduction}

RELAP5-3D was originally developed for analysis of light water reactors. The code does not represent axial or radial heat conduction in the fluid because the relatively low thermal conductivity of water ensures that their effects are small. However, the thermal conductivity of sodium is about 100 times greater than that of water. Consequently, heat conduction in the fluid has the potential to be important in fast reactors cooled by liquid sodium.

Kayes and Crawford (1980) present a simple rule of thumb that states that axial conduction may affect the heat transfer if the Peclet number is less than 100. The axial Peclet number for the XX09 subassembly in the EBR-II (Messick et al 1987) was about 100 at design conditions. Thus, the effects of axial conduction in the fluid could be important for fast reactors cooled by sodium, particularly during transients in which the flow decreases.

Yoo et al. (2003) evaluated the effects of fluid axial conduction in the proposed STAR-LM reactor, which is cooled by the natural circulation of lead-bismuth. They determined the relative importance of the axial conduction term by choosing reference parameters and obtaining a non-dimensional energy equation, which can be written as

$$
\frac{\mathrm{DT}^{+}}{\mathrm{Dt}^{+}}-\frac{1}{\mathrm{Pe}^{*}} \frac{\partial^{2} \mathrm{~T}^{+}}{\partial \mathrm{x}^{+2}}=\mathrm{Q}_{\mathrm{w}}^{+}
$$

where $\mathrm{T}$ is the temperature, $\mathrm{t}$ is time, $\mathrm{x}$ is the spatial coordinate, and the superscript + refers to a nondimensional parameter. The $\mathrm{Q}_{\mathrm{w}}$ term represents wall heat transfer, the details of which are not important here. The modified Peclet number, Pe*, can be written as

$$
\mathrm{Pe}^{*}=\operatorname{RePr} \frac{\mathrm{L}}{\mathrm{D}_{\mathrm{h}}}
$$

where Re is the Reynolds number, which is based on the hydraulic diameter, $\mathrm{D}_{\mathrm{h}}$, Pr is the Prandtl number, and $\mathrm{L}$ is the length of the component. Since the traditional Peclet number is the Reynolds number times the Prandtl number, the modified Peclet number is just the traditional value times the length-to-diameter ratio.

Appropriate choices for the reference parameters result in the first and third terms in Equation 1 having an order of magnitude of one at steady state. If the modified Peclet number is of order one, the axial conduction term will also be of order one and hence will be of comparable importance to the convection term. If the modified Peclet number is 100 , the axial conduction term will be roughly $1 \%$ of the convection term. Since RELAP5-3D should be able to represent second- and third-order effects, Equation 1 indicates that axial conduction in the fluid should be modeled when the modified Peclet number is less than 100 . 
A numerical method was developed to simulate the effects of axial conduction in the fluid. Figure 5 illustrates a simple nodalization that contains three control volumes and two junctions and defines a global distance coordinate, $\mathrm{x}$. The subscripts, $\mathrm{m}-1, \mathrm{~m}$, and $\mathrm{m}+1$ refer to the volumes while the subscripts $\mathrm{m}-1 / 2$ and $\mathrm{m}+1 / 2$ refer to the connecting junctions. The temperature, $\mathrm{T}$, at the center of each volume is assumed to be known. The density, $\rho$, heat capacity, $\mathrm{C}$, and thermal conductivity, $\mathrm{k}$, are assumed to be constant within a control volume, but are allowed to vary with temperature between control volumes. The geometry is defined by the length, $\Delta x$, and flow area, $\mathrm{A}$, of each volume.

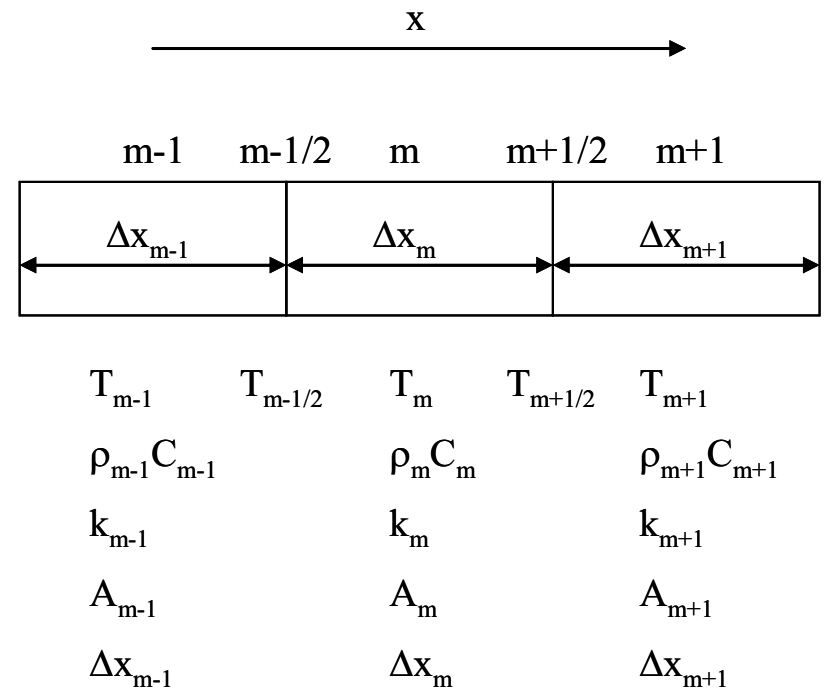

Figure 5. Nodalization diagram for the axial fluid heat conduction model.

Applying Fourier's law of heat conduction to calculate the heat transfer, q, between Volume m-1 and Junction $\mathrm{m}-1 / 2$ yields

$\mathrm{q}_{\mathrm{m}-1, \mathrm{~m}-1 / 2}=-\mathrm{k}_{\mathrm{m}-1} \mathrm{~A}_{\mathrm{m}-1} \frac{\mathrm{T}_{\mathrm{m}-1 / 2}-\mathrm{T}_{\mathrm{m}-1}}{0.5 \Delta \mathrm{x}_{\mathrm{m}-1}}$.

Similarly, the heat transfer from Junction $m-1 / 2$ to Volume $m$ is

$\mathrm{q}_{\mathrm{m}-1 / 2, \mathrm{~m}}=-\mathrm{k}_{\mathrm{m}} \mathrm{A}_{\mathrm{m}} \frac{\mathrm{T}_{\mathrm{m}}-\mathrm{T}_{\mathrm{m}-1 / 2}}{0.5 \Delta \mathrm{x}_{\mathrm{m}}}$.

For convenience, a volume property, B, and a junction property, D, are defined as

$\mathrm{B}_{\mathrm{m}}=\frac{\mathrm{k}_{\mathrm{m}} \mathrm{A}_{\mathrm{m}}}{\Delta \mathrm{x}_{\mathrm{m}}}$

and

$D_{m-1, m}=\frac{-2 B_{m-1} B_{m}}{B_{m-1} B_{m}}$.

No energy is assumed to be stored at the junction between adjacent volumes. Therefore, Equations 4 and 5 can be equated and solved to obtain the temperature at the junction 


$$
T_{m-1 / 2}=\frac{B_{m-1} T_{m-1}+B_{m} T_{m}}{B_{m-1}+B_{m}}
$$

Equations 5, 6, and 7 can be combined with either Equation 3 or 4 to obtain the heat transfer from Volume m-1 to Volume $\mathrm{m}$

$$
\mathrm{q}_{\mathrm{m}-1, \mathrm{~m}}=\mathrm{D}_{\mathrm{m}-1, \mathrm{~m}}\left(\mathrm{~T}_{\mathrm{m}}-\mathrm{T}_{\mathrm{m}-1}\right)
$$

Similar equations can be written for a more complicated case in which multiple junctions are attached to the inlet face of Volume $\mathrm{m}$ as shown in Figure 6. For this case, the heat transfer from Volume $\mathrm{j}$ to Volume m can be written as

$$
q_{j, m}=-2 B_{j}\left(\frac{B_{m}\left(T_{m}-T_{j}\right)+\sum_{i} B_{i} T_{i}-T_{j} \sum_{i} B_{i}}{B_{m}+\sum_{i} B_{i}}\right)
$$

where $j$ corresponds to one of the volumes attached to the inlet face of Volume $m(j=1,2$, or 3 in Figure 6 ) and the summations are performed over all the volumes connected to the inlet face. The heat transfer from all of the volumes connected at Junction $\mathrm{m}-1 / 2$ to Volume $\mathrm{m}$ can be obtained by summing Equation 9 over all the volumes connected to the inlet face of Volume $\mathrm{m}$

$$
\mathrm{q}_{\mathrm{m}-1 / 2, \mathrm{~m}}=\sum_{\mathrm{j}} \mathrm{q}_{\mathrm{j}, \mathrm{m}}
$$

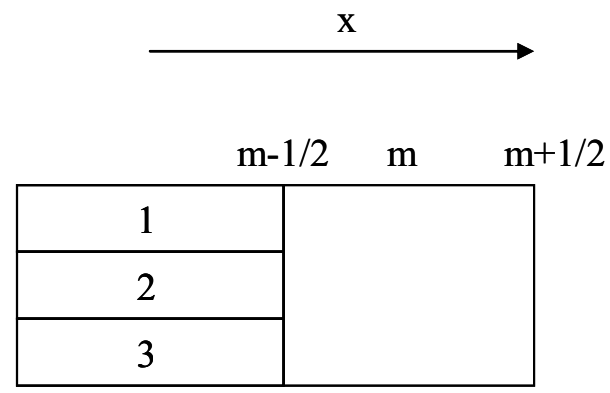

Figure 6. Nodalization diagram for the axial fluid heat conduction model with multiple junctions attached to the inlet face of a volume.

A similar derivation yields

$$
q_{m, j}=-2 B_{j}\left(\frac{B_{m}\left(T_{j}-T_{m}\right)-\sum_{i} B_{i} T_{i}+T_{j} \sum_{i} B_{i}}{B_{m}+\sum_{i} B_{i}}\right)
$$

for the case of multiple junctions attached to the outlet face of Volume $\mathrm{m}$.

The total heat added to Volume $\mathrm{m}, \mathrm{Q}_{\mathrm{m}}$, can be written as 


$$
\mathrm{Q}_{\mathrm{m}}=\sum_{\text {Inlets }} \mathrm{q}_{\mathrm{i}, \mathrm{m}}-\sum_{\text {Outlets }} \mathrm{q}_{\mathrm{m}, \mathrm{i}}
$$

where the first sum is taken over all the junctions attached to the inlet face of Volume $m$ and the second sum is taken over all the junctions attached to the outlet face of Volume $\mathrm{m}$. The usage of the terms "inlets" and "outlets" here is governed by the global distance coordinate, $\mathrm{x}$. The inlets are those junctions connected to the face of Volume $\mathrm{m}$ with the lower value of $\mathrm{x}$ while the outlets are connected to the face with the higher value of $\mathrm{x}$. Hence, the definition of inlets and outlets used here differs from the normal RELAP5-3D usage, where inlets and outlets are based on the definition of positive flow according to a local coordinate that can vary for every control volume.

The RELAP5-3D control system model summarized by Equation 12 can be extended to simulate radial conduction by using appropriate values for the geometry. The length term in Equation 5 is based on the input length in the crossflow direction. The flow area term in Equation 5 is calculated as the fluid volume

of the control volume divided by the input length. Since the fluid volume accounts for the presence of the fuel rods, the radial conduction model also accounts for the presence of the fuel rods in an average sense. A conduction shape factor, such as that described by Jeong et al. 2007, could be applied to obtain a more accurate representation of the geometrical effects on the radial heat conduction process, but was not used in this evaluation. Only one junction is connected to each radial face. Thus, the modifications for multiple connections shown in Equations 9 and 11 are not required for the radial conduction model.

\subsection{Radial Mixing}

Each fuel rod in the XX09 subassembly was wrapped with a helical wire that maintained separation between the fuel rods and promoted mixing between subchannels. A model was developed to simulate the effects of mixing between adjacent rings in the two-dimensional model summarized in Figure 4.

The calculated mixing between rings was based on the model developed by Cheng and Todreas (1986). The transfer of energy between adjacent subchannels is based on the dimensionless effective eddy diffusivity, $\varepsilon^{*}$,

$\varepsilon^{*}=$ effective transverse mass flux / axial mass flux $=\mathrm{C}_{\mathrm{m}}\left(\mathrm{A}_{\mathrm{rl}} / \mathrm{A}_{1}^{\prime}\right)^{1 / 2} \tan \theta$

where

$$
\mathrm{C}_{\mathrm{m}}=\mathrm{C}_{\mathrm{mT}}=0.14\left(\frac{\mathrm{P}-\mathrm{D}}{\mathrm{D}}\right)^{-0.5}
$$

for turbulent flow and

$$
\mathrm{C}_{\mathrm{m}}=\mathrm{C}_{\mathrm{mL}}=0.077\left(\frac{\mathrm{P}-\mathrm{D}}{\mathrm{D}}\right)^{-0.5}
$$

for laminar flow. The geometrical parameters $\mathrm{A}_{\mathrm{r} 1}, \mathrm{~A}_{1}^{\prime}$, and $\theta$ are calculated as

$$
\mathrm{A}_{\mathrm{r} 1}=\pi\left(\mathrm{D}+\mathrm{D}_{\mathrm{w}}\right) \mathrm{D}_{\mathrm{w}} / 6
$$




$$
\mathrm{A}_{1}^{\prime}=(\sqrt{3} / 4) \mathrm{P}^{2}-\pi \mathrm{D}^{2} / 8
$$

and

$$
\cos \theta=\mathrm{H} / \sqrt{\mathrm{H}^{2}+\left(\pi\left(\mathrm{D}+\mathrm{D}_{\mathrm{w}}\right)\right)^{2}}
$$

where $\mathrm{D}$ is the diameter of the fuel rod, $\mathrm{D}_{\mathrm{w}}$ is the diameter of the wire, $\mathrm{P}$ is the fuel rod pitch, and $\mathrm{H}$ is the height of one revolution of the helical wire wrap.

The flow regime is determined from the Reynolds number, Re, which is calculated for flow in the axial direction and accounts for the presence of the wire on the fluid velocity and the hydraulic diameter. For laminar flow,

$$
\operatorname{Re}<\operatorname{Re}_{\mathrm{L}}=10^{1.7(\mathrm{P} / \mathrm{D}-1)+\log _{10} 300} .
$$

For turbulent flow,

$$
\operatorname{Re}>\operatorname{Re}_{\mathrm{T}}=10^{0.7(\mathrm{P} / \mathrm{D}-1)+4} .
$$

The dimensionless effective eddy diffusivity in the transition region between laminar and turbulent flow is calculated as

$$
\varepsilon^{*}=\varepsilon_{\mathrm{L}}^{*}+\left(\varepsilon{ }_{\mathrm{T}}-\varepsilon_{\mathrm{L}}{ }_{\mathrm{L}}\right) \psi^{2 / 3}
$$

where

$$
\psi=\frac{\log _{10} \operatorname{Re}-\log _{10} \mathrm{Re}_{\mathrm{L}}}{\log _{10} \mathrm{Re}_{\mathrm{T}}-\log _{10} \mathrm{Re}_{\mathrm{L}}} .
$$

The dimensionless effective eddy diffusivity accounts for the mixing caused by the wire wrapping and turbulence. The mixing caused by the wire wrapping is larger than that caused by turbulence (Cheng and Todreas 1986). Based on the geometry of the XX09 subassembly, $\varepsilon^{*}$ varies between 0.013 for laminar flow and 0.023 for turbulent flow.

The effective transverse mass flow, $\dot{\mathrm{m}}_{\mathrm{T}}$, between rings is calculated as

$$
\dot{\mathrm{m}}_{\mathrm{T}}=\varepsilon * \mathrm{GA}_{\mathrm{T}}
$$

where $\mathrm{G}$ is the axial mass flux and $\mathrm{A}_{\mathrm{T}}$ is the transverse flow area, which is calculated as

$$
\mathrm{A}_{\mathrm{T}}=\mathrm{n}_{\text {rod }}(\mathrm{P}-\mathrm{D}) \Delta \mathrm{x},
$$

where $\mathrm{n}_{\text {rod }}$ is the number of rods along the boundary between rings and $\Delta \mathrm{x}$ is the height of the control volume.

The effective energy interchange, $Q_{i j}$, between rings $i$ and $j$ is calculated as 
$\mathrm{Q}_{\mathrm{ij}}=\dot{\mathrm{m}}_{\mathrm{T}} \mathrm{C}_{\mathrm{p}}\left(\mathrm{T}_{\mathrm{i}}-\mathrm{T}_{\mathrm{j}}\right)$

where $\mathrm{C}_{\mathrm{p}}$ is the specific heat capacity of the fluid and $\mathrm{T}$ is the fluid temperature. The values of the dimensionless effective eddy diffusivity and mass flux in Equation 23 and the heat capacity in Equation 25 are averaged from the corresponding values in the adjacent rings.

\section{CONDUCTION AND MIXING RESULTS}

\subsection{Axial Conduction}

The one-dimensional RELAP5-3D model shown in Figure 3 was used determine the effects of axial conduction in the fluid for a wide range of steady-state conditions and a loss-of-flow transient. The RELAP5-3D control system was used to calculate the heat transfer given by Equation 12 for all of the control volumes in the model except for the two time-dependent volumes. The model required about 7 control variables per junction to simulate axial conduction. The net power added to each volume due to axial conduction was input into the fluid using the direct heating option of the heat structures that represented the subassembly and thimble walls. Note that the net power could be either positive or negative.

A series of steady-state calculations was performed to determine the importance of axial conduction in the fluid for five operating conditions. The first operating condition corresponded to the design condition for the XX09, which was $2.594 \mathrm{~kg} / \mathrm{s}$ and $486 \mathrm{~kW}$ (Messick et al. 1987). The inlet temperature was $616 \mathrm{~K}$ based on the reported value for loss-of-flow tests in EBR-II (Mohr et al. 1987) and was held constant for all the calculations. The other four operating conditions were performed at reduced flows and powers. The design flow and power were multiplied by the same percentage, which varied between $10 \%$ and $0.01 \%$, so that the same steady outlet temperature would be achieved in each case.

The flow and power boundary conditions used are illustrated in Figure 7. The model initially started with no flow and no power. The flow was ramped up to the design value over $10 \mathrm{~s}$. The power was ramped up to the design value beginning at $10 \mathrm{~s}$ and ending at $20 \mathrm{~s}$. The calculation was then continued with constant boundary conditions until steady conditions were achieved. The flow and power were then decreased by an order of magnitude in a near step change until a new steady state was achieved. This process was continued until a steady state was obtained at all of the desired operating conditions with and without axial conduction in the fluid. Figure 8 shows that the modified Peclet number at the center of the core varied from about $10^{4}$ to 1 during this process. The figure also shows that axial conduction did not significantly affect the modified Peclet number. 


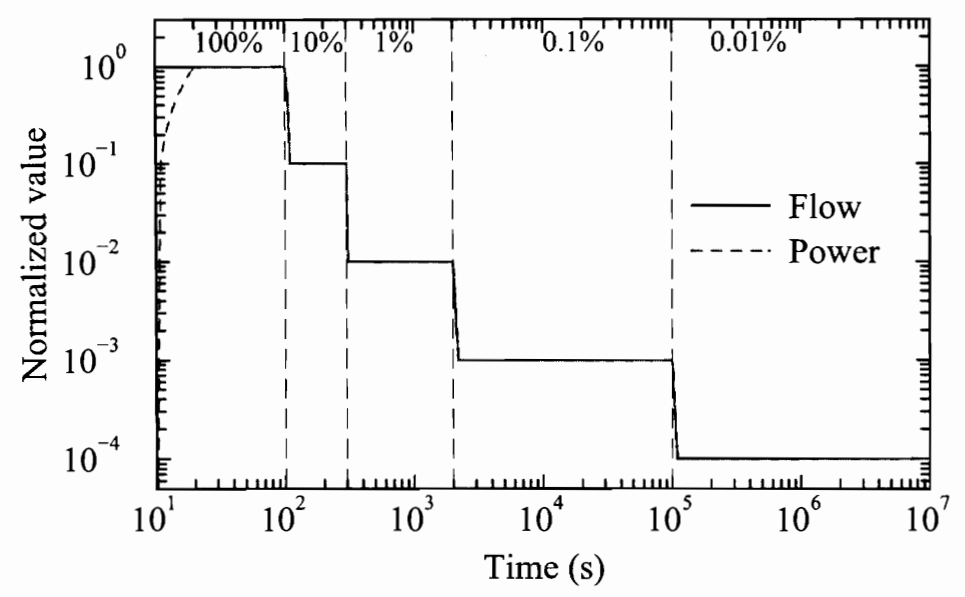

Figure 7. Flow and power boundary conditions used in the steady-state calculations.

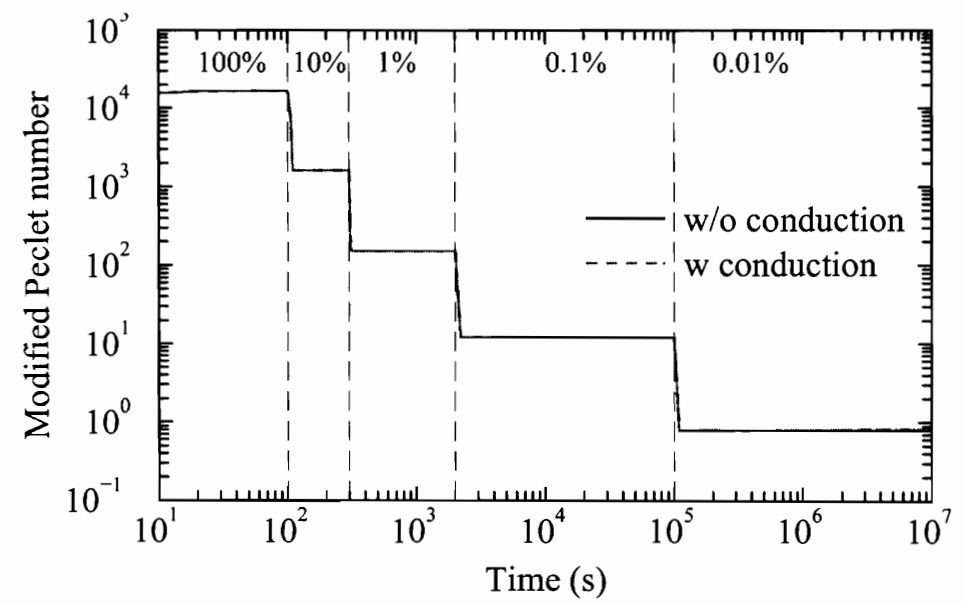

Figure 8. Modified Peclet numbers at the center of the core in the steady-state calculations.

Figure 9 shows steady-state axial fluid temperature profiles for the cases without axial conduction. As expected, the exit temperature was nearly the same for all of the cases because the power-to-flow ratio was the same for all the calculations. The axial temperature profiles were also nearly identical for all of the cases. 


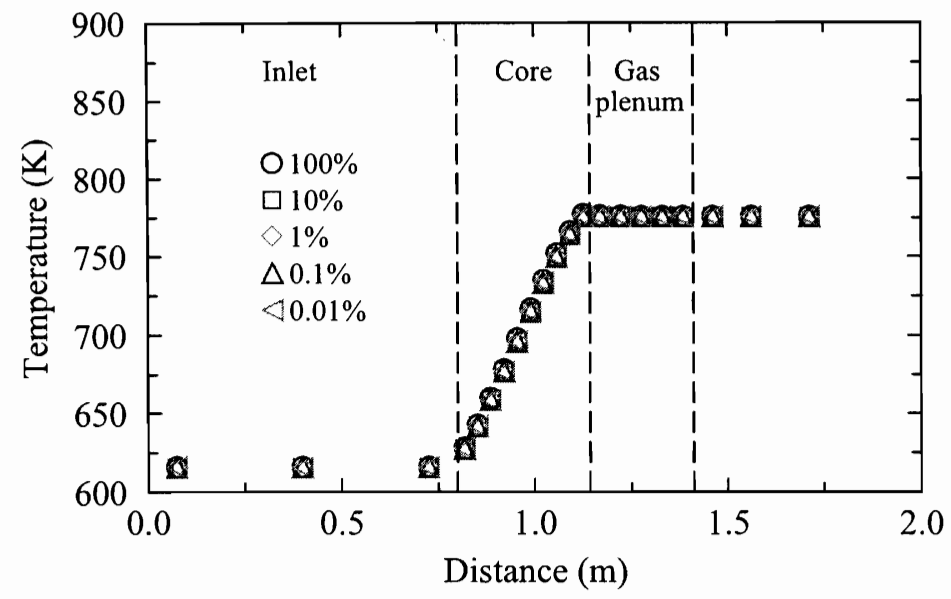

Figure 9. Axial fluid temperature profiles for the cases without axial conduction.

Figure 10 shows the steady-state axial fluid temperature profiles for the cases with axial conduction. The primary effect of the axial conduction was to preheat the fluid in the inlet region, which resulted in higher temperatures in the lower core. The amount of the preheating depended on the magnitude of the flow. The maximum difference in fluid temperature due to axial conduction was $0.08 \mathrm{~K}$ for the $100 \%$ case, 0.3 $\mathrm{K}$ for the $10 \%$ case, $2 \mathrm{~K}$ for the $1 \%$ case, $18 \mathrm{~K}$ for the $0.1 \%$ case, and $95 \mathrm{~K}$ for the $0.01 \%$ case. Based on a comparison of Figures 9 and 10, the effects of axial conduction were nearly imperceptible until the normalized flow decreased to $0.1 \%$.

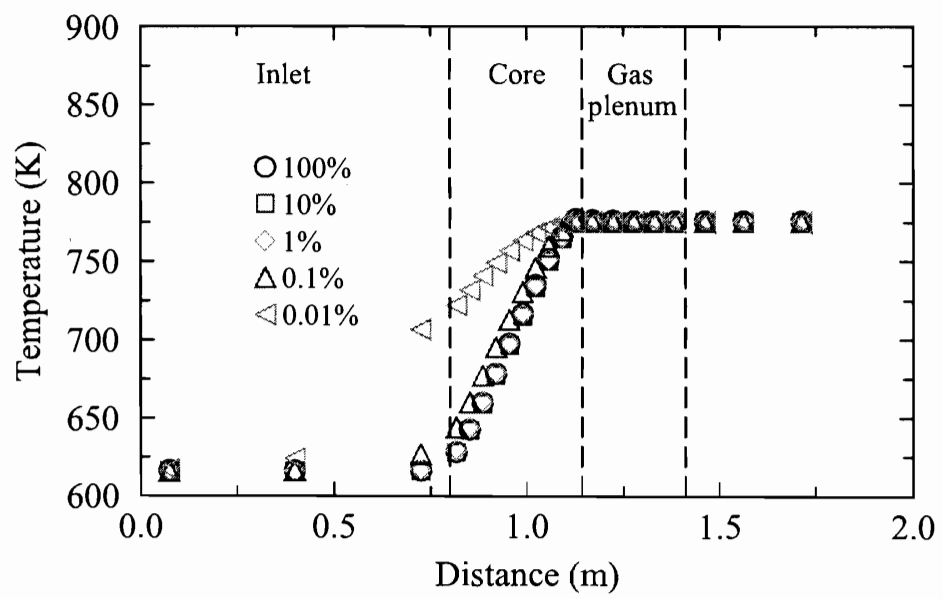

Figure 10. Axial fluid temperature profiles for the cases with axial conduction in the fluid.

The calculated effects of axial conduction shown by a comparison of Figures 9 and 10 are consistent with those expected from Equation 1. For example, the RELAP5-3D calculations indicate that the effects of axial conduction are nearly imperceptible in the $1 \%$ case. Figure 8 shows that the modified Peclet number for the $1 \%$ case was about 150 . Equation 1 indicates that the axial conduction term is expected to be about $0.7 \%$ of the convective term at a modified Peclet number of 100 and thus should be negligible. The RELAP5-3D calculations show that the effects of axial conduction are important for the $0.01 \%$ case, which had a modified Peclet number of 0.8 . Equation 1 indicates that the axial conduction term should be slightly larger than the convective term at a modified Peclet number of 0.8 , and thus clearly should be important. 
The results shown in Figure 10 accounted for axial conduction in the fluid, but did not account for axial conduction in the heat structures. A review of the geometry in the core indicated that the effects of axial conduction in the fluid should be larger than those due to axial conduction through the fuel rods because the area for axial conduction in the rods is about the same as that in the coolant and the thermal conductivity of the sodium is significantly greater than the average value for the rods. The effects of axial conduction of the heat structures were quantified using the code's conduction enclosure model, which allows an approximate multi-dimensional analytical capability because the user can input a conductance that simulates axial heat transfer between adjacent heat structures. The conduction enclosure model complements the code's one-dimensional heat conduction solution, which was applied in the radial direction for the EBR-II XX09 model. The enclosure model allows surfaces of heat structures to be thermally connected, but does not allow each radial node in the heat structure to communicate with adjacent nodes in the axial direction and thus is not truly two-dimensional. Nonetheless, important twodimensional effects can generally be captured (Oh et al. 2006, Paik 1999).

Conduction enclosure models were developed to represent axial conduction in the fuel rods and the subassembly and thimble walls. Figure 11 shows the steady-state axial fluid temperature profiles for cases with axial conduction in the fluid and heat structures. Axial conduction in the heat structures raised the temperature near the bottom of the core by up to $12 \mathrm{~K}$ compared to the calculations with axial conduction in the fluid only. As reported previously, axial conduction in the fluid alone raised fluid temperatures near the bottom of the core by $95 \mathrm{~K}$ compared to the case with no axial conduction. As expected, the effects of axial conduction in the heat structures are less important than those due to conduction in the fluid for the EBR-II XX09 subassembly.

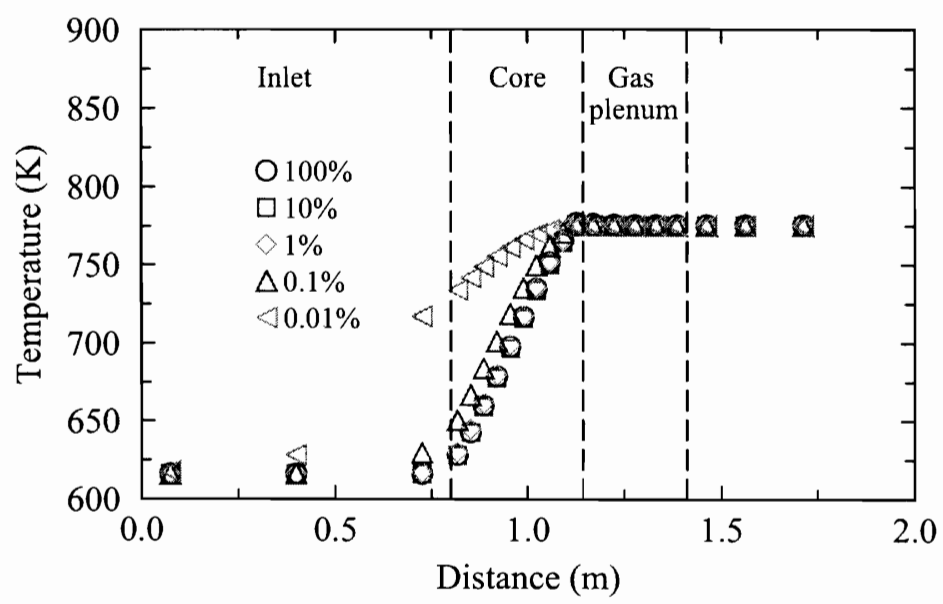

Figure 11. Axial fluid temperature profiles for the cases with axial conduction in the fluid and the heat structures.

The calculated results shown previously represented the effects of axial conduction for a wide range of steady-state conditions. Calculations were also performed that simulated a loss-of-flow transient in a loop-type reactor based on one described by Dunn and Prohammer (1982). The transient was initiated by a loss of the primary sodium pumps, which caused a reactor scram. After the primary pumps coasted down, the pony motors held the flow at about $15 \%$ until they were assumed to fail about 5 minutes into the event. Dunn and Prohammer reported that the flow reversed in most of the core channels before the onset of natural circulation. The flow in the high-powered subassembly never reversed, but approached stagnation conditions. 
The RELAP5-3D model shown in Figure 3 was used to simulate a loss-of-flow event based on the transient described by Dunn and Prohammer (1982). The normalized flow and power boundary conditions for the transient are shown in Figure 12. The inlet flow was assumed to completely stagnate for $40 \mathrm{~s}$ to maximize the effects of axial conduction. The power was assumed to remain constant at $2.5 \%$ of the design value for the XX09 subassembly, which is reasonably consistent with the expected decay power following infinite operation of U-235 (American Nuclear Society 1979). The normalized power exceeded the normalized flow for about $80 \mathrm{~s}$ prior to the onset of natural circulation near $440 \mathrm{~s}$. The inlet fluid temperature was held constant at $616 \mathrm{~K}$.

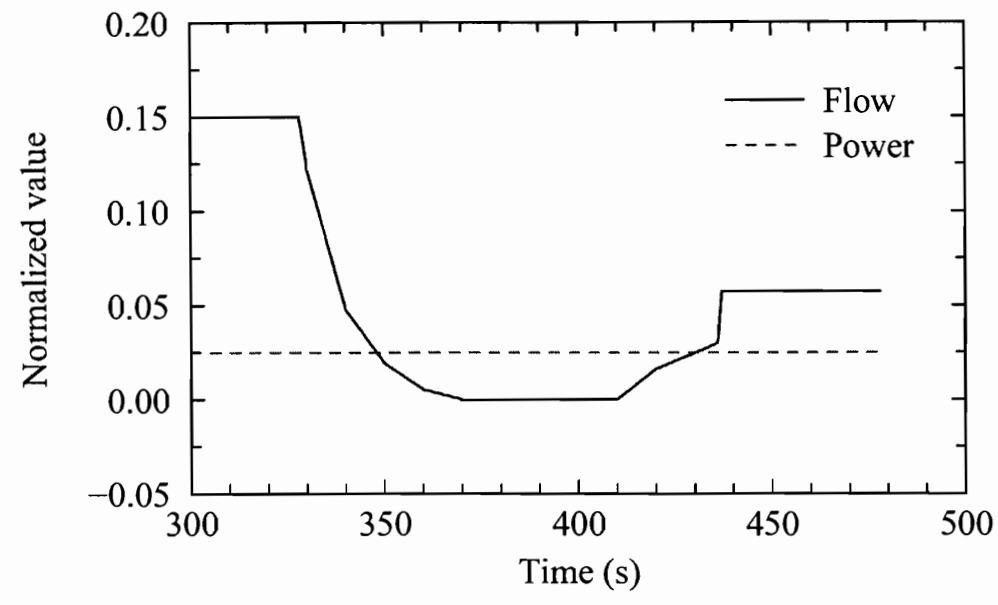

Figure 12. Boundary conditions for the loss-of-flow transient.

Three calculations were performed to simulate the loss-of-flow transient after the loss of the pony motor. The calculations were started from a steady state based on the normalized flow and power shown in Figure 12 at $300 \mathrm{~s}$. The first calculation neglected the effects of axial conduction in the fluid and the heat structures. The second calculation, which was the most mechanistic one, accounted for the effects of axial conduction using the control system summarized by Equation 12 to simulate the effects of conduction in the fluid and the enclosure models to simulate the effects of conduction in the heat structures. The third calculation did not use the control system to explicitly represent axial conduction in the fluid, but increased the conductances in the enclosure models to account for the combined effects of axial conduction in the fluid and the heat structures. The third calculation required two additional assumptions compared to the second calculation. First, the axial temperature gradient in the heat structure was assumed to be the same as in the adjacent fluid, which is a reasonable assumption at low power. Second, the thermal conductivity of the sodium was assumed to be constant because the enclosure model uses a constant conductance. The third calculation also used artificial heat structures in the inlet and outlet regions to carry the heat associated with fluid conduction. The mass of the artificial heat structures was kept small to limit the stored energy. The method used in the third calculation was evaluated as an approximate alternative to the use of the control system to represent axial conduction in the fluid.

The results of the three transient simulations are summarized in Figures 13 and 14, which show the maximum cladding temperature in the core as a function of time and the axial fluid temperature profile at $430 \mathrm{~s}$, respectively. Axial conduction raised the temperature at the bottom of the core and lowered the temperature at the top of the core, but the effects were relatively small. The largest difference in maximum cladding temperature was $16 \mathrm{~K}$. This difference slightly exceeds the $15 \mathrm{~K}$ value used to determine the onset of significance for peak cladding temperature. However, the effect of axial conduction was exaggerated by the assumption of complete flow stagnation at the inlet to the subassembly and by the use of the one-dimensional model, which does not allow internal natural 
circulation within the subassembly. As will be shown later, internal natural circulation is an important cooling mechanism at low flows.

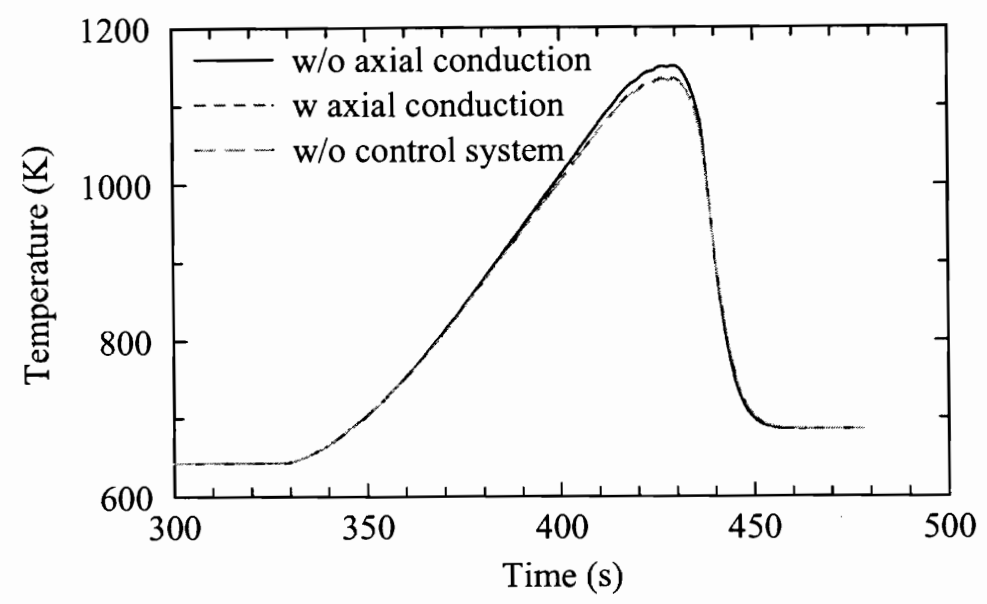

Figure 13. Maximum cladding temperature during the loss-of-flow transient.

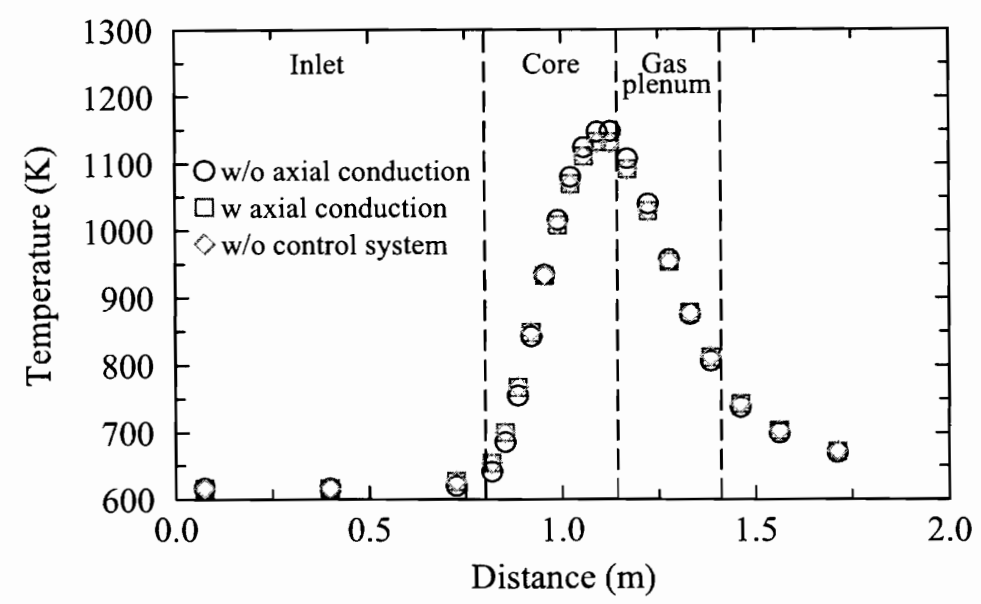

Figure 14. Axial fluid temperature profiles during the loss-of-flow transient.

A review of the calculated results showed that natural circulation between the subassembly and the thimble resulted in a small flow in the subassembly even when the inlet flow was zero. The natural circulation flow caused the minimum modified Peclet number in the subassembly to be about 10 during the transient and thus had the potential to suppress the effects of axial conduction in the fluid.

Consequently, the model was revised to prevent the natural circulation flow by deleting the junction connecting the inlet and thimble regions. The calculated effects of axial conduction on the maximum cladding temperature with the revised model were slightly smaller than those shown in Figure 13. The minimum modified Peclet number at the center of the subassembly was still about 2 because of the flow caused by thermal expansion during the transient.

Carslaw and Jaeger (1959) present a closed-form solution for one-dimensional transient heat conduction in a solid with constant thermal properties that is bounded by two parallel planes. Graphical results are presented for a solid that is at a uniform initial temperature and subjected to a step change in temperature at both surfaces, which are also applicable for the case in which the temperature at one surface 
experiences a step change and an artificial surface at the midpoint of the solid is adiabatic. Based on the geometry of the XX09 subassembly and the thermal properties of sodium, $190 \mathrm{~s}$ are required for $5 \%$ of the step change in temperature at one end of the core to reach the other end. The time required for $95 \%$ of the step change to propagate through the core is more than $2500 \mathrm{~s}$. Since the duration of flow stagnation in the transient was only $40 \mathrm{~s}$, it is not surprising that the effects of axial conduction are small during the transient.

A direct examination of the energy equation also confirms the fact that the axial conduction term is small compared to the transient term during the flow stagnation. The one-dimensional energy equation can be written as

$\rho C \frac{\partial \mathrm{T}}{\partial \mathrm{t}}+\rho \mathrm{Cv} \frac{\partial \mathrm{T}}{\mathrm{dx}}-\mathrm{k} \frac{\partial^{2} \mathrm{~T}}{\partial \mathrm{x}^{2}}=\mathrm{Q}_{\mathrm{w}}$

where $v$ is the fluid velocity, $\mathrm{Q}_{\mathrm{w}}$ is the heat per unit volume that is added to the coolant from the walls, and the other variables are as defined in Section 3. An evaluation of Equation 26 based on the geometry of the XX09 subassembly showed that the first and last terms were dominant during the flow stagnation and that the second and third terms were more than an order of magnitude smaller. Consequently, the effect of axial conduction on the peak cladding temperature is small compared to the temperature rise during the transient.

The two methods for modeling axial conduction in the fluid were essentially equivalent, with a maximum temperature difference of $0.4 \mathrm{~K}$. The method used in the third calculation was also used to repeat the steady-state calculations shown in Figure 11. The steady-state results from the two methods were also in excellent agreement, with a maximum difference of about $1.5 \mathrm{~K}$, which corresponds to about $1 \%$ of the maximum effect of axial conduction. Thus, the effects of axial conduction in the fluid can be adequately represented using only heat structures. However, this method is not preferred because it is not as mechanistic and requires significantly more work on the part of the user because the effects of axial conduction in the fluid have to first be calculated according to the method summarized in Equation 12 and then translated into equivalent input in the conduction enclosure models.

In summary, the effects of axial conduction in the fluid were generally not important. The steady-state calculations showed that axial conduction did not noticeably affect the fluid temperature profile in the core until the normalized power and flow decreased to about $0.1 \%$ of the design values. The effects of axial conduction were large when the normalized power and flow were $0.01 \%$ of the design values. Even though the normalized flow during the loss-of-flow event was less than $0.1 \%$ of the design value for more than $40 \mathrm{~s}$, transient terms dominated and axial conduction did not significantly affect the peak cladding temperature or the fluid temperature profile in the core. The effects of axial conduction in the fuel rods and other heat structures were smaller than those due to axial conduction in the fluid.

\subsection{Radial Heat Transport}

Radial fluid temperature measurements showed variations of nearly $100 \mathrm{~K}$ near the top of the EBR-II XX09 subassembly (Messick et al. 1987) during normal operation. Consequently, evaluations were performed to determine the importance of radial heat transport due to conduction and mixing. The evaluations utilized the two-dimensional RELAP5-3D model shown in Figure 4.

Calculations were performed both with and without the radial heat transport models summarized by Equations 12 and 25 for the sixty crossflow junctions illustrated in Figure 4 in the core and gas plenum regions. The radial conduction and mixing models required about 8 and 15 control variables per junction, respectively. Artificial heat structures were added to the inner four rings of the model so that the net 
power added to each volume due to radial heat transport could be accounted for using the code's direct moderator heating option. The artificial heat structures, which were only used in the calculations with radial conduction and radial mixing, were small. Their thermal capacitance was only $1 \%$ of that of the cladding in the corresponding control volume, so that they would not excessively affect calculated results during transients. The effects of axial conduction in the fluid and heat structures were neglected. The calculations that used the radial conduction model did not utilize the radial mixing model and vice versa so that the influences of each heat transport mechanism could be distinguished.

The five steady-state calculations described previously in the evaluation of axial conduction were repeated with the two-dimensional RELAP5-3D model. Figure 15 shows calculated radial temperature profiles at the top of the core for normalized flows and powers varying between $100 \%$ and $0.01 \%$ of the design values. These results were obtained without radial conduction in the fluid or radial mixing. The radial variation in temperature was large for the $100 \%$ and $10 \%$ cases, with a maximum value of about $130 \mathrm{~K}$ between the first and last rings. The large radial variation in fluid temperature was primarily caused by differences in the power-to-flow ratios. The mass flow rate in each ring was nearly proportional to the flow area of the ring. In the inner four rings, the power-to-flow ratio was relatively high because the fluid was heated by fuel rods on both the inner and outer boundaries. In the outermost ring, the power-to-flow ratio was about half of that in the inner rings because the subassembly wall was unheated. Cooling of the outermost ring by the flow through the thimble region also contributed to the radial temperature variation. The radial temperature profiles were nearly flat when the normalized power and flow were $1 \%$ or less of the design values. For these cases, buoyancy enhanced the flow through the interior rings and reduced the flow in the outermost ring, which flattened the radial temperature profile. For the $100 \%$ and $10 \%$ cases, buoyancy effects were insignificant compared to the frictional effects.

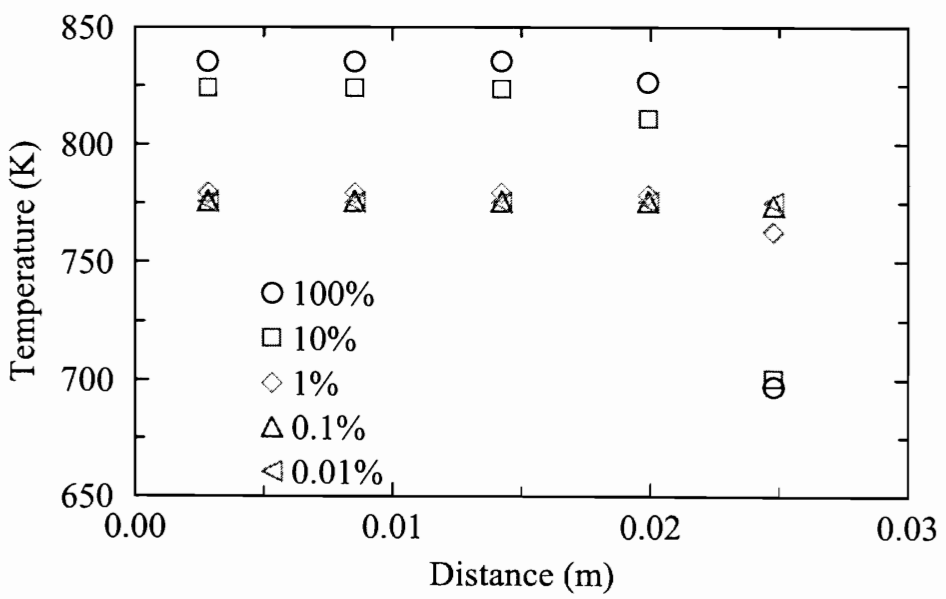

Figure 15. Steady-state radial fluid temperature profiles at the top of the core (without radial heat transport).

Figure 16 shows calculated steady-state fluid temperature profiles at the top of the core with the radial conduction model activated. The effect of radial conduction on the maximum fluid temperature, which occurred in the first ring, was less than $5 \mathrm{~K}$ for all the cases evaluated. However, radial conduction had a much larger effect on the temperature profile when the normalized flows and powers were $10 \%$ or greater. The temperature profiles were relatively flat when the normalized flows and powers were $1 \%$ or lower, which was similar to the results shown previously without radial conduction. 


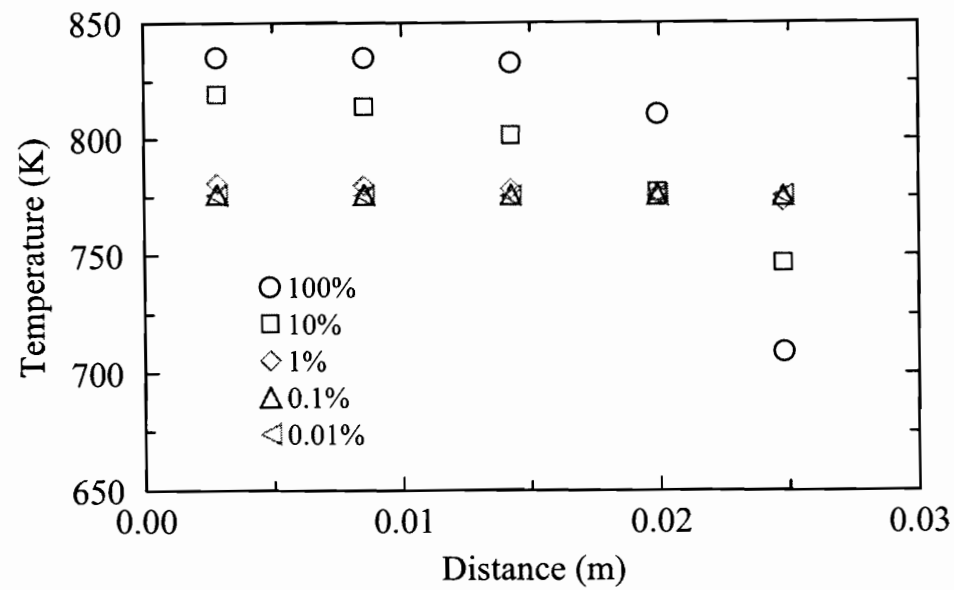

Figure 16. Steady-state radial fluid temperature profiles at the top of the core (with radial conduction).

Figure 17 shows calculated radial temperature profiles at the top of the core for normalized flows and powers varying between $100 \%$ and $0.01 \%$ of the design values with the radial mixing model turned on. These results were obtained without the radial fluid conduction model. The results are qualitatively similar to those reported previously with radial conduction in the fluid.

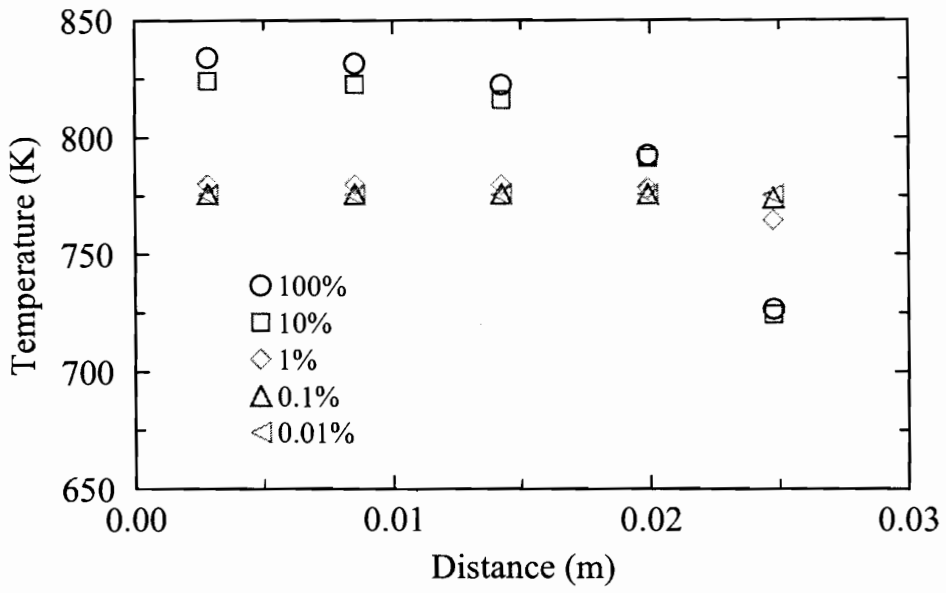

Figure 17. Steady-state radial fluid temperature profiles at the top of the core (with radial mixing).

The relative effects of the various models are compared in Figures 18 through 20. Figures 18 and 19 show that the maximum fluid temperature was nearly the same for all calculations with the twodimensional model when the normalized flow and power were $10 \%$ or more. However, radial conduction in the fluid and radial mixing between rings flattened the temperature profile. These radial heat transport mechanisms reduced the temperature in the fourth ring and increased the temperature in the fifth ring. The effective conductance for radial conduction $\left(-\mathrm{D}_{\mathrm{j}}\right.$ in Equation 6$)$ was about four times bigger than the corresponding value for radial mixing $\left(\dot{\mathrm{m}}_{\mathrm{T}} \mathrm{C}_{\mathrm{p}}\right.$ in Equation 25$)$ at $100 \%$ power and flow. Consequently, radial mixing had a larger effect on the temperature profile than radial conduction at full power and flow. However, the effect on the temperature profile was reversed at $10 \%$ power and flow, with conduction more important than mixing. The relative importance of the mixing term decreased because the effect of mixing is roughly proportional to the axial flow, which was a factor of 10 lower in the $10 \%$ case. The conduction and mixing terms did not significantly affect the temperature profile when the normalized 
power and flow were less than or equal to $1 \%$ because the energy transport within the subassembly was dominated by internal natural circulation.

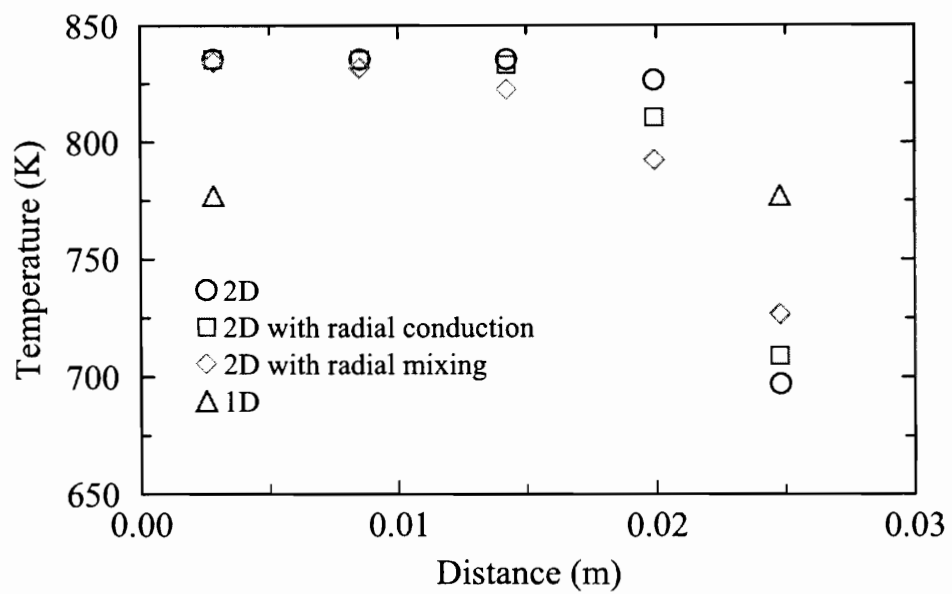

Figure 18. The effects of various models on steady-state radial fluid temperature profiles at the top of the core for a normalized flow and power of $100 \%$.

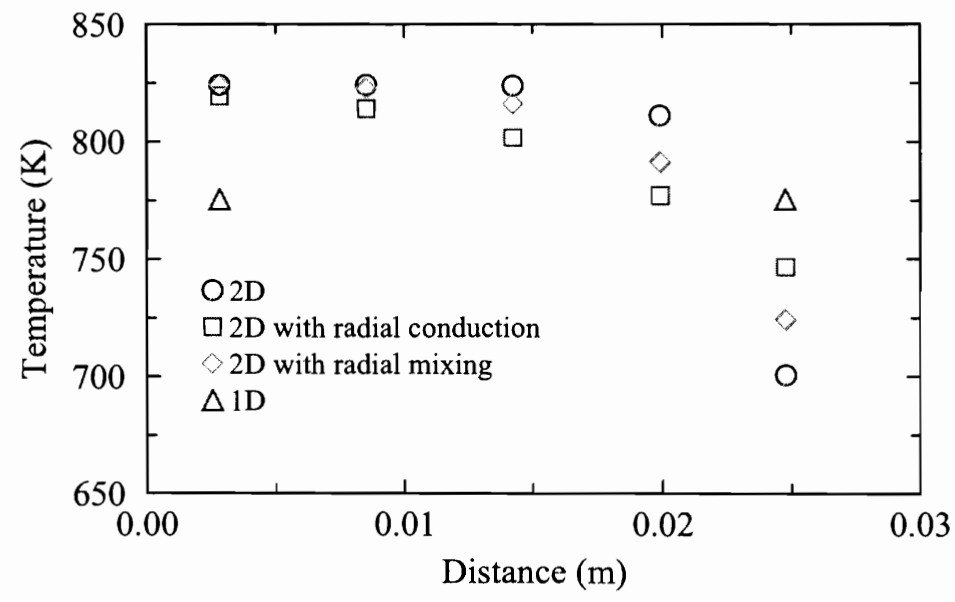

Figure 19. The effects of various models on steady-state radial fluid temperature profiles at the top of the core for a normalized flow and power of $10 \%$. 


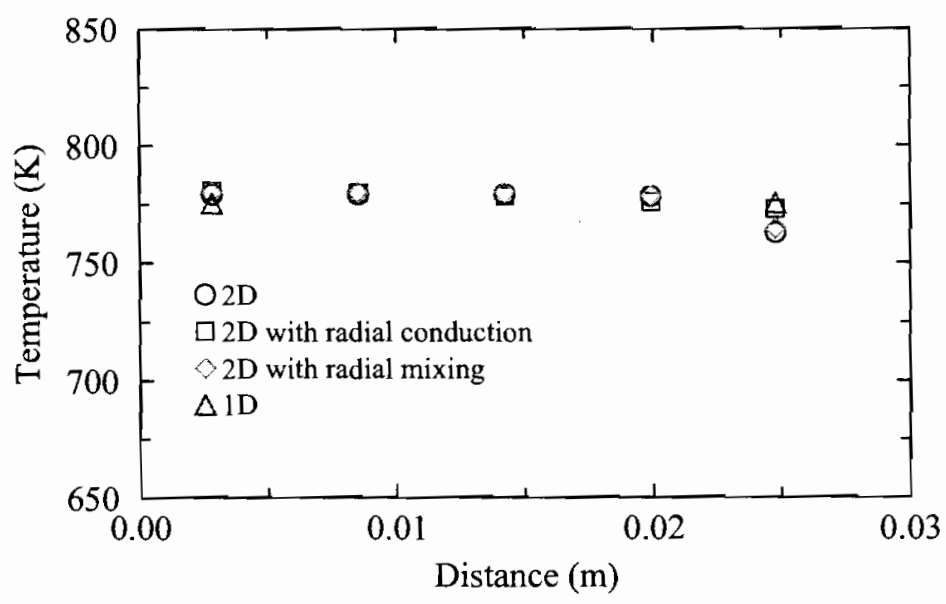

Figure 20. The effects of various models on steady-state radial fluid temperature profiles at the top of the core for a normalized flow and power of $1 \%$.

Figures 18 and 19 show that the one-dimensional model underpredicted the maximum fluid temperature for the $100 \%$ and $10 \%$ cases by about $50 \mathrm{~K}$. Thus, the one-dimensional model would also underpredict the maximum cladding temperature by about $50 \mathrm{~K}$ during normal operation. The one-dimensional model provides a good estimate of the maximum cladding temperature at the lower flow rates when the temperature profile is relatively flat, but of course does not account for the natural circulation within the subassembly.

Figures 21 and 22 show the relative effects of the various models on the axial fluid velocity profiles at the top of the core. All the two-dimensional models predicted a relatively flat velocity profile at $100 \%$ flow, with the largest velocity occurring in the outermost ring because of its larger hydraulic diameter. These results indicate that the velocity profile during normal operation is dominated by friction effects. The two-dimensional model without radial conduction and mixing predicted much steeper radial gradients when the normalized power and flow were $1 \%$ of the design values. In this case, the velocity profile was dominated by buoyancy effects. The velocity was lowest in the outermost ring, which was heated by only one row of rods instead of two rows for the inner rings. Figure 22 shows that radial heat conduction flattened the velocity profile significantly, but the effect of radial mixing was small.

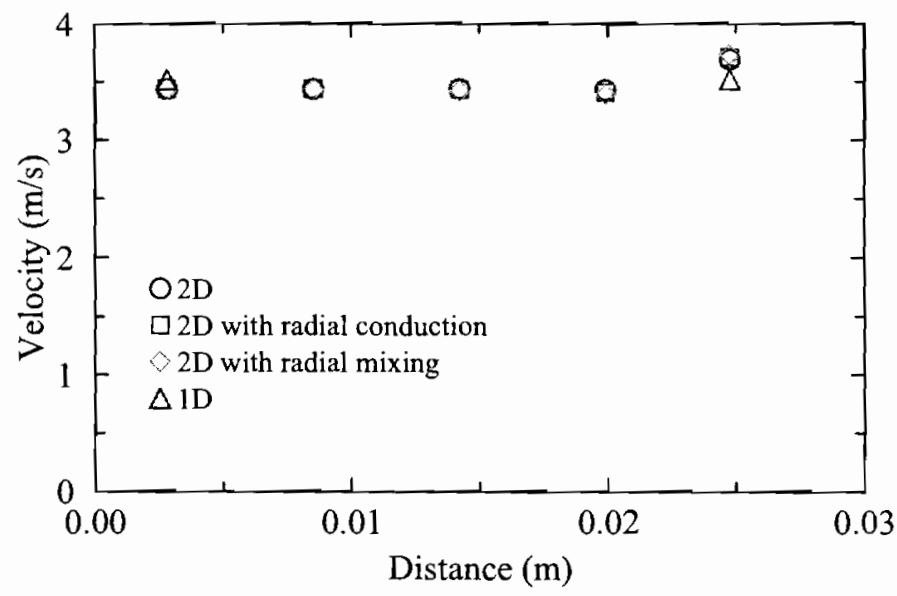

Figure 21. The effects of various models on steady-state axial fluid velocity profiles at the top of the core for a normalized flow and power of $100 \%$ case. 


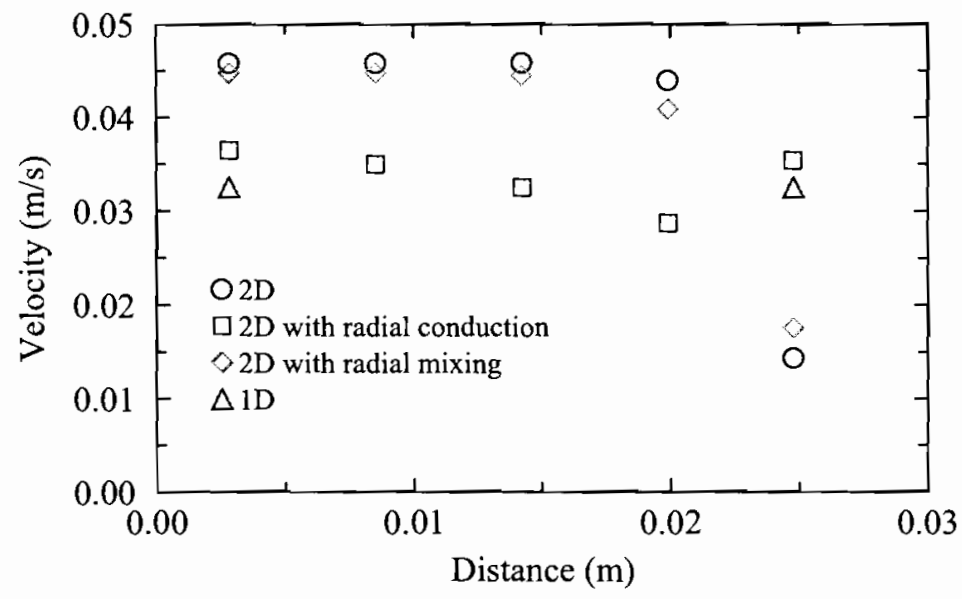

Figure 22. The effects of various models on steady-state axial velocity profiles at the top of the core for a normalized flow and power of $1 \%$ case.

A loss-of-flow transient based on the one described by Dunn and Prohammer (1982) was simulated using the two-dimensional RELAP5-3D model summarized in Figure 4. Figure 23 shows the effect of the radial conduction and mixing models on the maximum cladding temperature in the core. The location of the maximum temperature varied during the transient, but was always located in the inner ring and in the upper third of the core. The figure also shows results from the one-dimensional model illustrated in Figure 3.

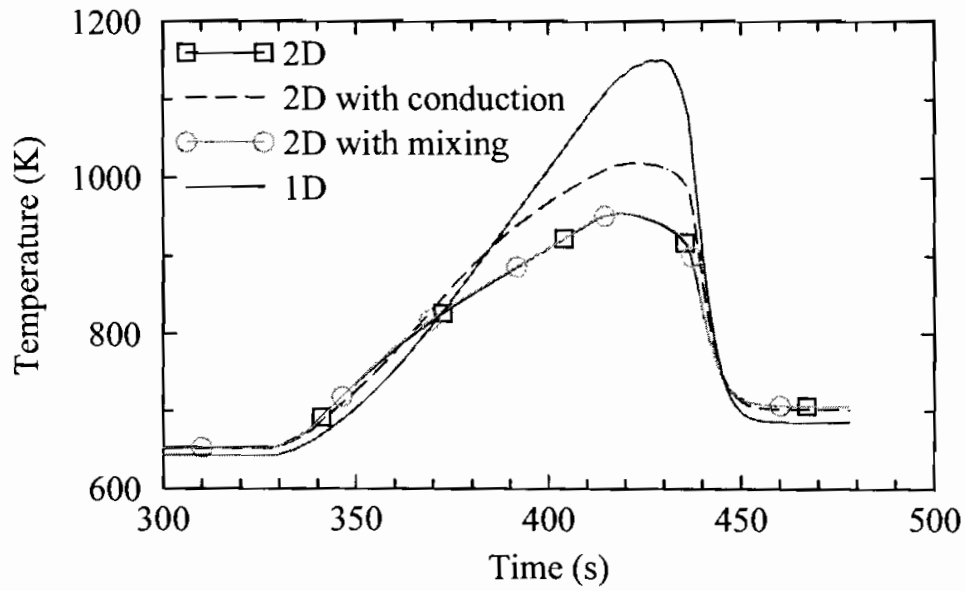

Figure 23. The effects of radial heat transport mechanisms on the maximum cladding temperature during the loss-of-flow transient.

The maximum cladding temperatures predicted by the two-dimensional models were about $10 \mathrm{~K}$ higher that from the one-dimensional model prior to the loss of the pony motor near $330 \mathrm{~s}$, but the onedimensional model predicted the highest cladding temperature during the transient by a large margin. The peak value predicted with the two-dimensional model without radial heat transport was $200 \mathrm{~K}$ lower than the one-dimensional result because of the mixing within the subassembly due to the buoyancy-driven flows. The higher flow rates with the two-dimensional models also resulted in cooler fluid reaching the top of the core sooner after the inlet flow restarted, which resulted in an earlier peak temperature. The effect of radial mixing was insignificant because the amount of mixing is proportional to the axial flow 
rate, which was relatively small during the loss-of-flow transient. The calculated peak cladding temperature with radial conduction in the fluid was $66 \mathrm{~K}$ higher than the calculation without radial conduction. Radial conduction by itself would tend to flatten the radial temperature profile, which should result in a lower peak cladding temperature during a transient. However, the flatter temperature profile also reduced the natural circulation flow inside the subassembly as shown by Figure 24. The reduced flow with radial conduction caused higher temperatures in the core and lower temperatures in the gas plenum. The flow was initially upwards through the entire core in all of the calculations. However, the flow in the outermost ring reversed shortly before and returned positive shortly after the inlet flow, shown previously in Figure 12, stagnated in the two-dimensional calculations. This reverse flow in the outermost ring allowed the gas plenum and core regions to mix during the flow stagnation and was responsible for the lower cladding temperatures with the two-dimensional models.

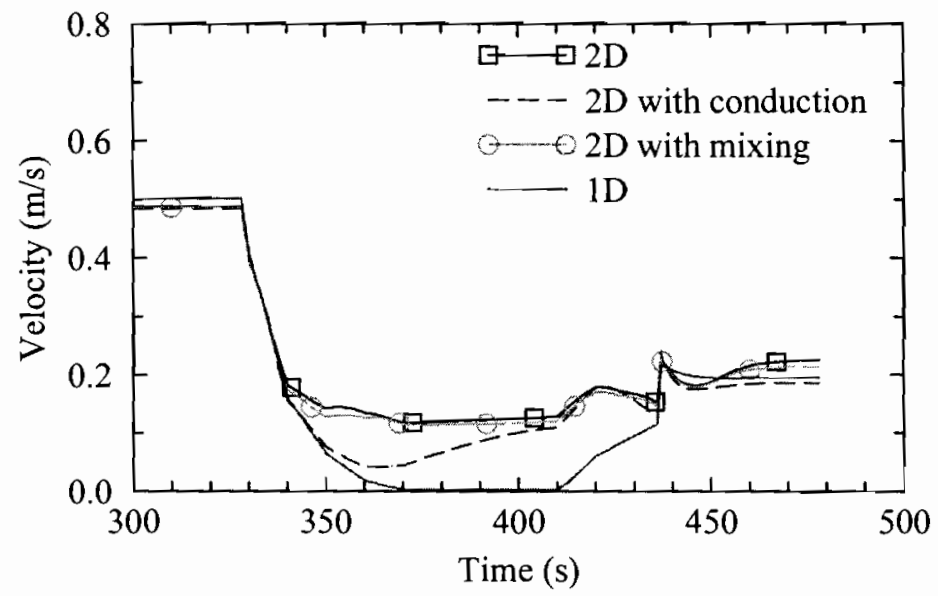

Figure 24. The effects of radial heat transport mechanisms on the axial fluid velocity at the top of the core in the third ring during the loss-of-flow transient.

Radial conduction in the fluid significantly affected the results during the loss-of-flow transient, which was in sharp contrast to the results obtained previously with axial conduction. The fact that radial conduction significantly affects transient results is consistent with theoretical considerations. Carslaw and Jaeger (1959) present a closed-form solution for the one-dimensional temperature distribution across a solid cylinder with a constant initial temperature that is subjected to a step change in the temperature at the outer surface for the case with constant thermal properties. The closed-form solution was applied to a solid cylinder with a radius equal to that of the XX09 subassembly and with heat transfer properties that are representative of sodium. The time required for $95 \%$ of the step change to propagate to the interior of the cylinder was only $7 \mathrm{~s}$. Since the duration of flow stagnation in the transient was $40 \mathrm{~s}$, it is not surprising that the effects of radial conduction were significant.

In summary, the effects of radial conduction and mixing in the fluid can be important. Although radial conduction and mixing did not significantly affect the maximum fluid temperature in the subassembly at steady state, they did significantly affect fluid temperatures near the periphery of the subassembly at high flow rates and powers. The effects of the radial heat transport models were relatively small when the normalized flow and power were $1 \%$ or less because of internal subassembly flow due to buoyancy. The two-dimensional model had a large effect on the peak clad temperature during the loss-of-flow transient. Radial heat conduction in the fluid also significantly affected the peak cladding temperature during the transient. 


\subsection{Stability}

The fluid conduction model summarized in Equation 12 utilizes the old-time temperature values and is an explicit numerical scheme. Explicit solutions to the heat conduction equation in solids have a numerical stability limit (Carslaw and Jaeger 1959) that can be represented as

$\Delta \mathrm{t} \leq \frac{\rho \mathrm{C} \Delta \mathrm{x}^{2}}{2 \mathrm{k}}$

where $\Delta t$ is the time step size. Based on the thermal properties of sodium and the node sizes for the XX09 subassembly in the RELAP5-3D model, the stability limit is about $10 \mathrm{~s}$ for axial conduction and $0.25 \mathrm{~s}$ for radial conduction. These values are greater than the time step size typically used with RELAP5-3D during transients. A finer nodalization than used here would reduce the maximum allowed time step size and thus could be of concern with respect to stability. However, there is a large margin with respect to the axial nodalization. The radial nodalization of one ring per row of rods used in this evaluation is probably the most detailed radial nodalization that would ever be used with RELAP5-3D. Thus, an explicit numerical scheme is expected to be acceptable for representing heat conduction in the fluid in RELAP5-3D.

\section{EM PUMP MODELING}

Two EM pump models were reviewed to determine how well they could be simulated using existing models in RELAP5-3D. The first model was a mechanistic model (Murray 2005) developed for an annular liquid induction pump based on equations described by Baker and Tessier (1987). The second model was based on a simple, linear equation.

The mechanistic model developed by Murray (2005) required 16 input parameters that described the geometry of the annular linear induction pump, electrical parameters, such as the frequency, voltage, number of poles and phases, and the temperature, which was used to determine physical properties of the liquid metal. The model then predicted pump operating parameters, such as differential pressure and efficiency, as a function of flow. Murray developed a model of the sodium EM pump of Araseki et al. (2000). Calculations were performed for a range of flows and electrical parameters varying between 15 and $20 \mathrm{~Hz}, 105$ and $224 \mathrm{~V}$, and 4 to 6 poles. The calculated results were in generally good agreement with the measured values.

The EM pump operating parameters reported by Murray (2005) were converted to equivalent operating parameters for the centrifugal pump model in RELAP5-3D. Murray reported a differential pressure that was calculated from the pressures at the outlet and inlet of the pump. The reported differential pressure thus included the head developed by the EM pump, frictional losses due to the wall and inlet/outlet effects, and the hydrostatic head. The RELAP5-3D centrifugal pump model assumes that the effects of wall friction are included within the pump performance curves, which is consistent with the results reported by Murray. However, the code explicitly accounts for hydrostatic effects. Consequently, the hydrostatic differential pressure was removed from the value reported by Murray so that the hydrostatic effects would not be counted twice. Because the resulting differential pressure includes frictional losses, wall friction should be turned off in the RELAP5-3D model (the code always turns wall friction off in pump components) and inlet and exit losses should not be input.

Murray (2005) defined the efficiency as the hydraulic power developed by the EM pump divided by the total power input. The differential pressure developed by the pump was calculated from the developed 
hydraulic power multiplied by a constant to account for the end effects based on the recommendation of Baker and Tessier (1987). The constant was 0.6 for the pump evaluated by Murray. Since the developed head for the EM pump was reduced by a factor of 0.6 to account for end effects, the efficiency of the equivalent centrifugal pump is 0.6 times the value reported by Murray.

The resulting differential pressure and efficiency for a centrifugal pump equivalent to the EM pump simulated by Murray (2005) are given as a function of flow in Figures 25 and 26 . The efficiency decreases rapidly as the flow approaches the synchronous value of $3.06 \mathrm{~m}^{3} / \mathrm{min}$.

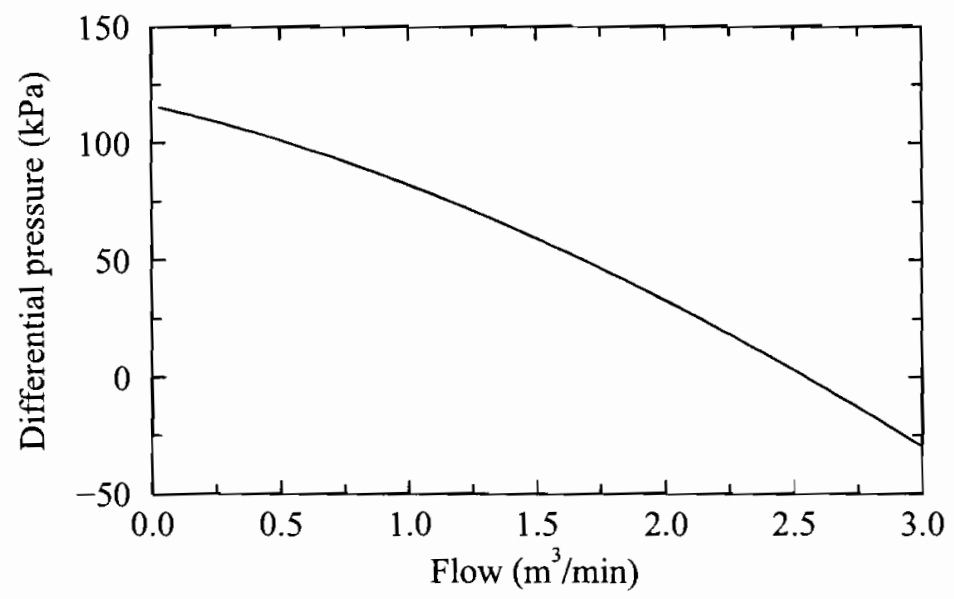

Figure 25. Differential pressure versus flow.

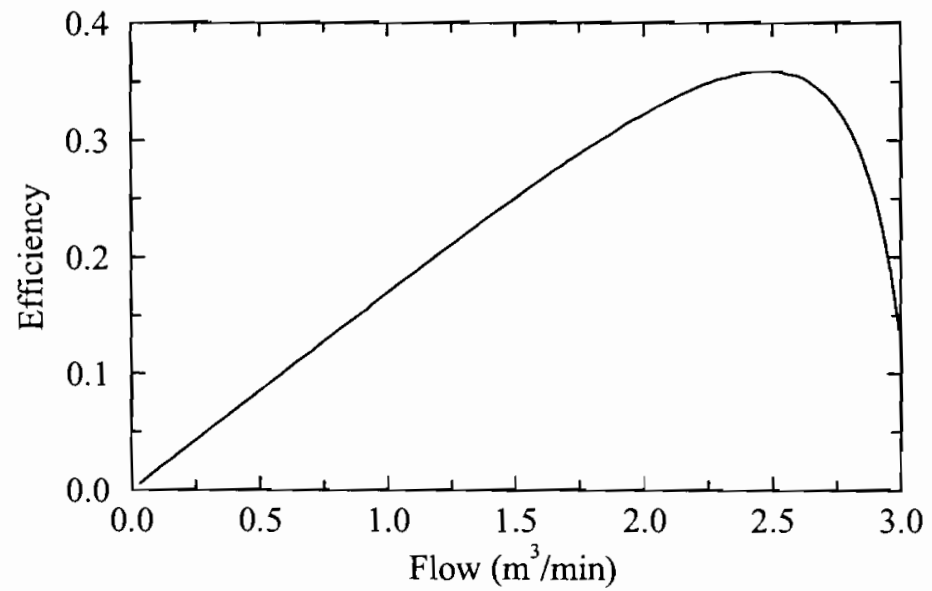

Figure 26. Efficiency versus flow.

The curves shown in Figures 25 and 26 were converted to the dimensionless homologous parameters utilized by the RELAP 5-3D centrifugal pump model. The homologous parameters require a definition of rated head, flow, and torque to normalize the various variables. Rated conditions are generally evaluated at the point of maximum efficiency, but the differential pressure for this pump is nearly zero at the point of maximum efficiency because the pressure generated by the pump is approximately balanced by the pressure lost due to friction. Consequently, the rated conditions for this pump were taken at a flow midway between zero and the flow corresponding to the point of maximum efficiency. Rated parameters are given in Table 1. 
Table 1. Rated parameters for the EM pump modeled by Murray (2005).

\begin{tabular}{|l|c|}
\hline Parameter & Value \\
\hline & \\
\hline Rated speed, $\mathrm{rad} / \mathrm{s}$ & 125.7 \\
\hline Rated flow, $\mathrm{m}^{3} / \mathrm{s}$ & 0.02035 \\
\hline Rated head, $\mathrm{m}$ & 8.259 \\
\hline Rated efficiency & 0.2064 \\
\hline Rated torque, $\mathrm{N}-\mathrm{m}$ & 57.0 \\
\hline Rated density, $\mathrm{kg} / \mathrm{m}^{3}$ & 897 \\
\hline
\end{tabular}

The homologous head and torque derived for the EM pump modeled by Murray (2005) are shown in Figures 27 and 28. The homologous head for the EM pump looks similar to that typically obtained for centrifugal pumps. The homologous torque also looks similar except near an independent value of 0.4 in Regime 2, where the dimensionless torque decreases rapidly as the efficiency heads towards zero at the synchronous flow rate.

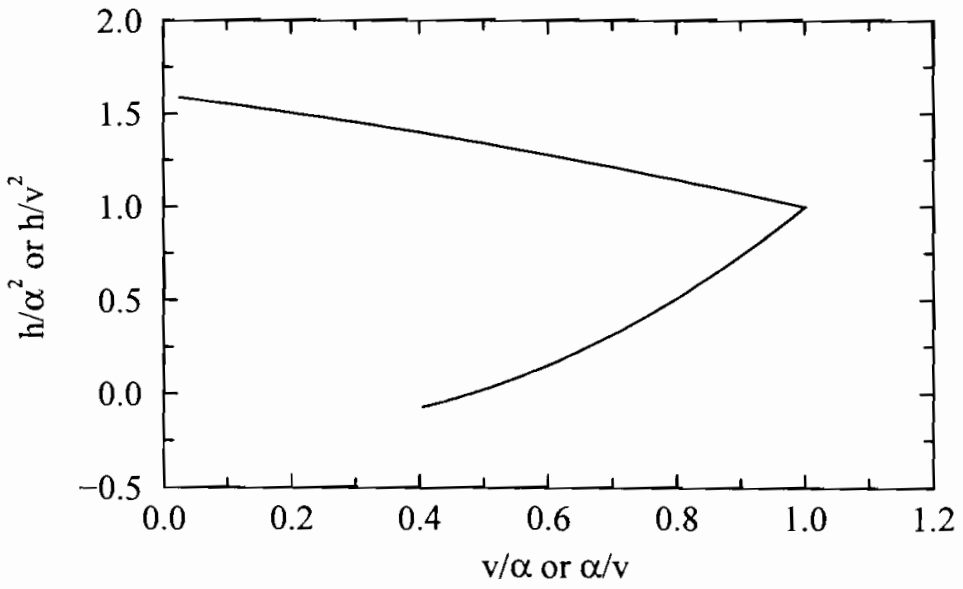

Figure 27. Homologous head for an EM pump.

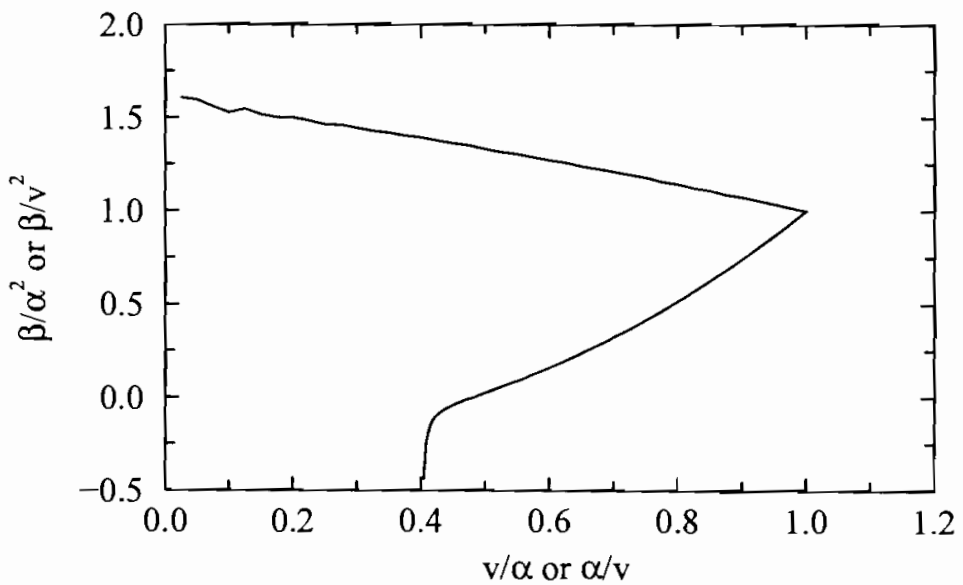

Figure 28. Homologous torque for an EM pump. 
The results shown in Figures 27 and 28 indicate that the centrifugal pump model used in RELAP5-3D should be able to adequately simulate the performance of an EM pump as a function flow with normal electrical parameters.

An alternate, but more complicated, approach would be to use the RELAP5-3D control system to explicitly represent Murray's model to calculate the head and efficiency of the EM pump. The speed of a surrogate centrifugal pump could then be adjusted to obtain the head produced by the EM pump. A heat structure with a heat source could be used to deposit the correct amount of energy into the fluid so that the same total energy was input to the system with both pumps. Although this approach would have the benefit of mechanistic modeling of the EM pump performance, it would be more complicated than using the homologous curves shown in Figures 27 and 28.

The centrifugal pump model was also compared with a simple model in which the head produced is assumed to be a linear function of flow that depends on the status of the power supply. The linear model is based on the stall head, which is defined as the head produced at zero flow. The stall head is assumed to be a function of the power supply, but the flow at zero head is assumed to be constant. The maximum value of the stall head occurs when $A C$ power is available and is used to normalize the stall head when AC power is not available. Degraded linear functions of flow are used following a loss of AC power. If no backup power supply is available, the stall head rapidly decreases to zero. If a backup power supply, such as a battery, is available, the stall head will degrade gradually with time as the battery discharges. For this evaluation, the degraded, normalized stall head was assumed to be 0.80 .

The linear model was simulated with the centrifugal pump model that is available in RELAP5-3D. Figure 29 compares results from the linear and centrifugal models for two cases, one where normal AC power remains available and the other where the produced head was degraded to simulate operation with a battery. The first case is referred to as "steady state" because the normalized speed in the centrifugal pump model remained constant at unity. The other case is referred to as "transient" because the normalized head at zero flow was decreased to simulate operation with a battery during a transient. The rated condition was assumed to be at a normalized flow rate of 0.95 on the steady-state curve. The results show that the desired function of head versus flow can be replicated exactly during steady-state operation in Regime 1, where the normalized flow is less than the rated value of 0.95 , and very closely in Regime 2 , where the normalized flow is greater than 0.95 . As a result, there are no perceptible differences between the two models during steady-state operation. To simulate the operation of the battery, the normalized centrifugal pump speed was reduced to the square root of the normalized stall head $(\sqrt{0.8}=0.894)$. The resulting head exactly matched the results from the linear model at zero flow. However, differences between the two models appeared as the flow increased. The maximum difference in the normalized head was about 0.1 and occurred near a normalized flow of 0.9 . A normalized speed of zero will provide the correct head after the battery is discharged.

Although the transient results obtained with the homologous model are probably good enough, more accurate results could be obtained by adjusting the pump speed. The pump speed needed to match the linear model can be calculated from a quadratic equation, which can be solved using the control system of RELAP5-3D. For example, increasing the pump speed from 0.894 to 0.966 at a normalized flow of 0.8 allows the homologous model to match the linear model during the transient. The use of the control system to calculate the desired performance of the EM pump and then solve the quadratic equation for the appropriate normalized speed as a function of the desired stall head and the flow rate would allow the homologous model to exactly match the linear model as a function of time. A steady-state value of the stall head will have to be obtained from a detailed model, such as that described by Murray (2005) or from another source, such as measurements. 


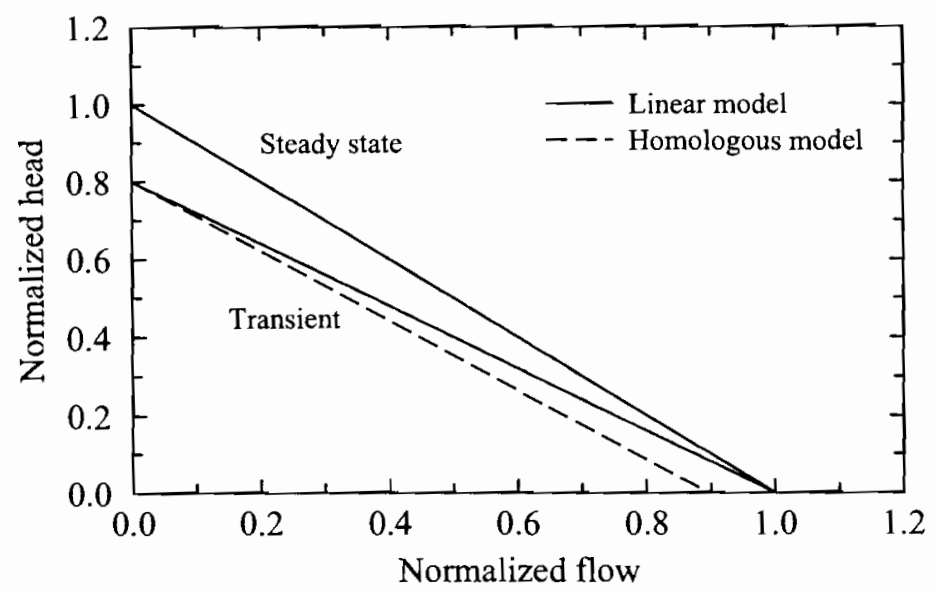

Figure 29. A comparison of linear and homologous EM pump models.

\section{CONCLUSIONS}

The effects of axial conduction in the fluid are not important for most ABTR applications. Axial conduction did not noticeably affect the steady-state temperature profiles until the normalized flow and power decreased to $0.1 \%$ of the design values. The calculated effects of axial conduction were large when the normalized power and flow were $0.01 \%$ of the design values. The effects of axial conduction during the loss-of-flow transient were relatively small because of the short time periods involved. Even though the calculated effects of axial conduction were relatively small, they were probably overstated because the analyses were performed with the one-dimensional model, which neglected the internal flows caused by buoyancy effects. If the two-dimensional model had been used, it is expected that the effects of internal natural circulation would have overwhelmed the effects of axial conduction when the normalized power and inlet flow were less than or equal to $1 \%$ of the design values.

Subchannel effects within the subassembly are important in the calculation of peak cladding temperature in the ABTR. The two-dimensional RELAP5-3D model predicted more than $100 \mathrm{~K}$ radial variations in fluid temperature at the top of the core during normal operation, primarily because the outermost ring was bounded by an unheated subassembly wall. The one-dimensional model underestimated the maximum fluid temperature in the subassembly by more than $50 \mathrm{~K}$ during normal operation. During a loss-of-flow transient, the one-dimensional model did not account for the internal recirculation due to buoyancy and thus neglected an important cooling mechanism. As a result, the peak cladding temperature obtained with the one-dimensional model was more than $130 \mathrm{~K}$ higher than the corresponding values from the twodimensional models.

The effects due to radial conduction in the fluid are also important in the calculation of peak cladding temperature for the ABTR. Radial conduction significantly affected the steady-state radial temperature profile when the normalized power and flow were $10 \%$ or more of the design values. Radial heat conduction increased the peak cladding temperature by about $60 \mathrm{~K}$ during a loss-of-flow transient.

The effects of radial mixing in a subassembly are also important at high flow rates. The steady-state analyses showed that the mixing between subassemblies affected the radial fluid temperature profile more than radial conduction did at design conditions. The effect of mixing on the peak cladding temperature during a loss-of-flow transient was small because the amount of mixing was proportional to the axial flow rate, which was small during the event. The effects of radial mixing are expected to be important during transients with high flow rates. 
Although RELAP5-3D does not have internal code models to represent heat conduction within a fluid or radial mixing between subchannels, both phenomena can be adequately simulated using the code's control system model. However, the control system approach has two limitations. First, this approach places a burden on the user in terms of the amount of work required to represent these phenomena. Internal code models that could be easily turned on or off would be far easier to use than developing a control system for each new application. Second, the control system approach places an upper limit on the number of junctions at which radial heat transport can be modeled. Because of the finite number of control variables available, radial conduction and mixing can be simulated for a maximum of about 430 junctions. Therefore, internal code models that calculate the effects of heat conduction and mixing in the fluid should be added to RELAP5-3D to support analyses of the ABTR.

The performance of an EM pump was adequately simulated with a centrifugal pump model. The homologous curves used in the centrifugal pump model adequately represented the performance predicted by a mechanistic EM pump model during normal operation. The homologous approach also reasonably simulated the performance of a simple, linear model during a transient. If necessary, more accurate results could be obtained during a transient by adjusting the pump speed with the control system.

\section{REFERENCES}

American Nuclear Society, 1979, Decay Heat Power in Light Water Reactors, ANSI/ANS-5.1.

Araseki, H., I. R. Kirillov, G. V. Preslitsky, A. P. Ogorodnikov, 2000, "Double Supply Frequency Pressure Pulsation In Annular Linear Induction Pump Part I: Measurement and Numerical Analysis," Nuclear Engineering and Design, Vol. 195, 85-100.

Baker and Tessier, 1987, Handbook of Electromagnetic Pump Technology, Chapter 7, Elsevier Science Publishing Company, New York.

Carslaw, H. S. and J. C. Jaeger, 1959, Conduction of Heat in Solids, Second Edition, Oxford University Press, London.

Cheng, S. K. and N. E. Todreas, 1986, "Hydrodynamic Models and Correlations for Bare and Wirewrapped Hexagonal Rod Bundles - Bundle Friction Factors, Subchannel Friction Factors and Mixing Parameters," Nuclear Engineering and Design, 92, pp. 227-251.

Davis, C. B., 2006, Applicability of RELAP5-3D for Thermal-Hydraulic Analyses of a Sodium-Cooled Actinide Burner Test Reactor, INL/EXT-06-11518, July.

Dunn, F. E., J. E. Cahalan, D. Hahn, and H. Jeong, 2006, "Whole Core Sub-Channel Analysis

Verification with the EBR-II SHRT-17 Test," Paper 6364, Proceedings of ICAAP'06, Reno NV, June 4-8, 2006.

Dunn, F. E. and F. G. Prohammer, 1982, SASSYS Analysis of Degraded Shut-Down Heat Removal Performance in LMFBRs, ASME Paper No. 82-WA/HT-37.

Jeong, H. Y., K. S. Ha, Y. M. Kwon, Y. B. Lee, D. Hahn, J. E. Cahalan, and F. E. Dunn, "Evaluation of the Conduction Shape Factor with a CFD Code for a Liquid-metal Heat Transfer in Heated Triangular Rod Bundles, Nuclear Engineering and Design, Article in Press, 2007. 
Kays, W. M. and M. E. Crawford, 1980, Convective Heat and Mass Transfer, Second Edition, McGrawHill Book Company, New York.

Lehto, W. K., R. M. Fryer, E. M. Dean, J. F. Koenig, L. K. Chang, D. Mohr, and E. E. Feldman, 1987, "Safety Analysis for the Loss-of-Flow and Loss-of-Heat Sink Without Scram Tests in EBR-II," Nuclear Engineering and Design, 101, pp. 35-44.

Messick, N. C., P. R. Betten, W. F. Booty, L. J. Christensen, R. M. Fryer, D. Mohr, H. P. Planchon, and W. H. Radtke, 1987, "Modification of EBR-Il Plant to Conduct Loss-of-Flow-without-SCRAM Tests," Nuclear Engineering and Design, Vol. 101, 13-23.

Mohr, D., L. K. Chang, E. E. Feldman, P. R. Betten, and H. P. Planchon, 1987, "Loss-of-Primary-Flow Without-Scram Tests: Pretest Predictions and Preliminary Results," Nuclear Engineering and Design, 101 , pp. 45-56.

Murray, P. E., 2005, An Electromagnetic Annular Liquid Induction Pump Model for RELAP5-3D, May, Enclosure 1 of INL Letter From J. W. Duling to K. Collins, "Electromagnetic Pump Model Design Report," CCN 201305, June 1, 2005.

NRC, 2007, Acceptance Criteria for Emergency Core Cooling Systems for Light-water Nuclear Power Reactors, Code of Federal Regulations, 50.46, http://www.nrc.gov/reading-rm/doccollections/cfr/part050/part050-0046.html.

Oh, C. H, C. Davis, L. Siefken, R. Moore, H. C. NO, J. Kim, G. C. Park, J. C. Lee, and W. R. Martin, 2006, Development of Safety Analysis Codes and Experimental Validation for a Very High Temperature Gas-Cooled Reactor, INL/EXT-06-01362, March.

RELAP5-3D Development Team, 2005, RELAP5-3D Code Manual Volume 1: Code Structure, System Models and Solution Methods, INEEL-EXT-98-00834, Revision 2.3, April.

Paik, S., 1999, "RELAP5-3D Multidimensional Heat Conduction Enclosure Model for RBMK Reactor Application," Nuclear Technology, Vol. 128, pp. 87-101, October.

Talley, J., 2006, "Modeling EBR-II Loss of Flow and Loss of Heat Sink without SCRAM using RELAP5-3D," 2006 International RELAP5 Users Seminar, West Yellowstone, MT, Sept. 16-18.

Yoo, Y. J., P. Sabharwall, J. N. Reyes, Q. Wu, and J. J. Sienicki, 2003, "Effects of Fluid Axial Conduction on Liquid Metal Natural Circulation and Linear Stability," 2003 ANS/ENS International Winter Meeting, pp. 1523-1530, (Global 2003), New Orleans, LA, November 16-20. 